\title{
Optima and Equilibria for a Model of Traffic Flow
}

\author{
Alberto Bressan and Ke Han \\ Department of Mathematics, Penn State University \\ University Park, Pa. 16802, U.S.A. \\ e-mails: bressan@math.psu.edu ,kxh323@psu.edu
}

August 9, 2011

\begin{abstract}
The paper is concerned with the Lighthill-Whitham model of traffic flow, where the density of cars is described by a scalar conservation law. A cost functional is introduced, depending on the departure time and on the arrival time of each driver. Under natural assumptions, we prove the existence of a unique globally optimal solution, minimizing the total cost to all drivers. This solution contains no shocks and can be explicitly described. We also prove the existence of a Nash equilibrium solution, where no driver can lower his individual cost by changing his own departure time. A characterization of the Nash solution is provided, establishing its uniqueness. Some explicit examples are worked out, comparing the costs of the optimal and the equilibrium solution. The analysis also yields a strategy for optimal toll pricing.
\end{abstract}

\section{Introduction}

Aim of this paper is to analyze globally optimal solutions and Nash equilibrium solutions for a specific problem of traffic flow. Car drivers starting from a location $A$ (a residential neighborhood) need to reach a destination $B$ (a working place) all at a given time $T$. There is a cost for starting early and a cost for arriving late. Clearly, these costs can also account for the total time spent in the car. Denoting by $\tau_{d}$ and $\tau_{a}$ respectively the departure and the arrival time, the total cost to an individual driver is

$$
\Psi \doteq \varphi\left(\tau_{d}\right)+\psi\left(\tau_{a}-T\right)
$$

An appropriate choice of the penalty functions here is

$$
\varphi(s)=-s, \quad \psi(s)=\left\{\begin{array}{cc}
0 & \text { if } s \leq 0 \\
\alpha s^{2} & \text { if } s \geq 0
\end{array}\right.
$$


If $L$ is the length of the highway connecting $A$ with $B$, and $v$ is the speed of cars, then $\tau_{a}=\tau_{d}+\frac{L}{v}$. It is now easy to compute the optimal departure time for each driver:

$$
\tau_{d}^{\mathrm{opt}}=\underset{s}{\operatorname{argmin}}\left\{\varphi(s)+\psi\left(s+\frac{L}{v}-T\right)\right\}
$$

The trouble is that, if everyone adopts the same optimal strategy and departs exactly at the same time, a big traffic jam is created and this strategy is not optimal anymore. To resolve this issue, one needs to look at a better model, taking into account the fact that the speed of cars depends on the traffic density.

Call $\rho=\rho(t, x)$ the density of cars at time $t$ at the point $x$ along the highway. The LighthillWitham-Richards model $[15,16]$ describes the evolution of $\rho$ in terms of the conservation law

$$
\rho_{t}+[\rho v(\rho)]_{x}=0 .
$$

Here the decreasing function $v=v(\rho)$ describes the velocity of cars depending on the density. A common choice is

$$
v(\rho)=a_{1} \ln \frac{a_{2}}{\rho} \quad 0 \leq \rho \leq a_{2} .
$$

The choice

$$
v(\rho)=\left(1-\frac{\rho}{\rho_{0}}\right) v_{0} \quad 0 \leq \rho \leq \rho_{0}
$$

is also meaningful. It yields the same qualitative properties and explicit solution formulas.

An optimization problem can now be formulated as follows. A planner needs to schedule departures, in such a way that the combined total cost is as small as possible. Let

$$
\rho(t, A) v(\rho(t, A))=\bar{u}(t)
$$

be the departure rate from $A$, i.e., how many drivers enter the highway per unit time. Notice that the boundary condition (1.6) at $x=A$ is meaningful provided that the characteristic speed $\partial_{\rho}(\rho v(\rho))$ is positive. As in the examples (1.4)-(1.5), we shall assume that the map $\rho \mapsto \rho v(\rho)$ is concave down, attaining a positive maximum at a point $\rho^{*}>0$. We regard $t \mapsto \bar{u}(t)$ as a control function. In other words, $\bar{u}(\cdot)$ is a measurable function that can be assigned at will, subject only to the constraint

$$
\bar{u}(t) \in[0, M], \quad M \doteq \max _{\rho \geq 0} \rho v(\rho) .
$$

Clearly, the incoming flux cannot be larger than the maximum flux allowed by the conservation law (1.3). The condition that all drivers eventually have to depart can be written as a constraint

$$
\int \bar{u}(t) d t=\kappa
$$

where $\kappa$ is the total number of drivers.

Let $\rho=\rho(t, x)$ be the solution of conservation law (1.3), defined for $(t, x) \in \mathbb{R} \times[A, B]$, with boundary data (1.6) assigned at $x=A$, and let

$$
u(t, x) \doteq \rho(t, x) v(\rho(t, x)) \quad t \geq 0, \quad x \in[A, B]
$$



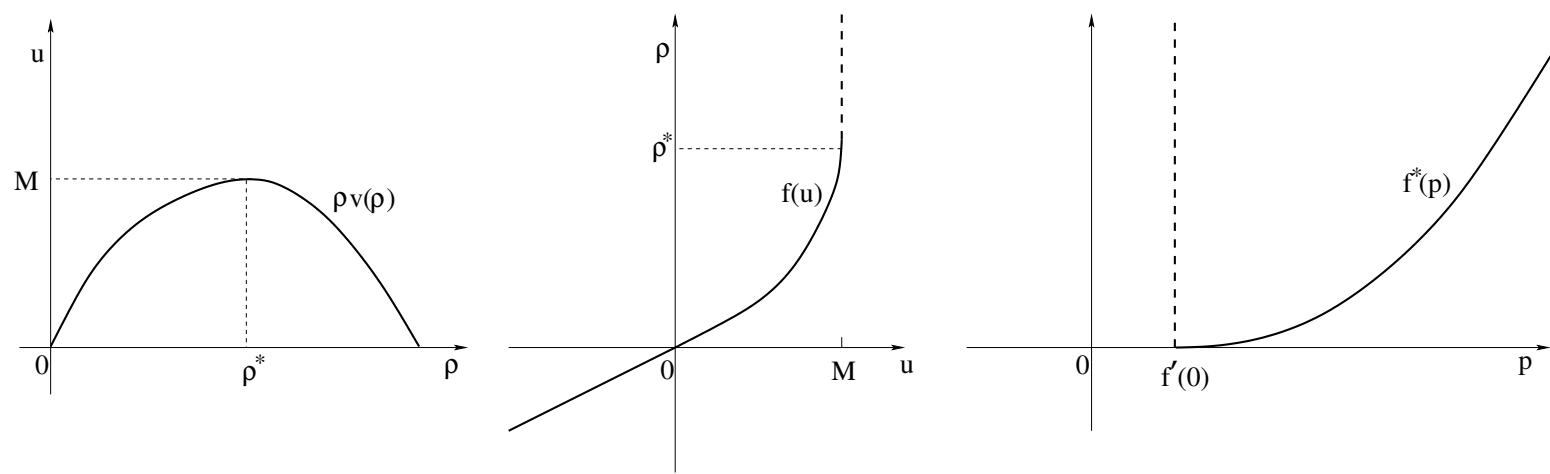

Figure 1: Left: the function $\rho \mapsto \rho v(\rho)$ describing the flux of cars. Middle: the function $f$, implicitly defined by $f(\rho v(\rho))=\rho$ and extended according to (2.9). Right: the Legendre transform $f^{*}$.

be the corresponding flux. The total cost is then measured by

$$
J(u)=\int \varphi(t) u(t, A) d t+\int \psi(t-T) u(t, B) d t .
$$

It is convenient to switch the roles of the variables $t, x$, replacing the boundary value problem (1.3)-(1.6) with a Cauchy problem for the conservation law describing the flux $u=\rho v(\rho)$, namely

$$
\begin{gathered}
u_{x}+f(u)_{t}=0, \\
u(t, 0)=\bar{u}(t) .
\end{gathered}
$$

The function $u \mapsto f(u)=\rho$ is defined as a partial inverse of the function $\rho \mapsto \rho v(\rho)=u$, (see Figure 1), assuming that

$$
0 \leq u \leq M \doteq \max _{\rho \geq 0} \rho v(\rho), \quad 0 \leq \rho \leq \rho^{*} \doteq \underset{\rho}{\operatorname{argmax}} \rho v(\rho) .
$$

Remark 1. We are here making an important modeling assumption. Namely, the car density never exceeds $\rho^{*}$, hence the characteristic speed is positive: $\frac{\partial}{\partial \rho}(\rho v(\rho)) \geq 0$. As time increases, this means that characteristics move from the boundary (where $x=A$ ) toward the interior of the domain. Notice that this assumption is consistent with a causality principle (fig. 2). By assigning the values of the incoming flux of cars at time $\tau$, if $\rho<\rho^{*}$ we influence the values of the solution at future times $t>\tau$. However, if $\rho>\rho^{*}$, this boundary data would influence the values of the solution in the past, at times $t<\tau$.

A solution to (1.10)-(1.11) can be explicitly determined using the Lax formula [13]. Adjusting the variables so that $T=0$ and $[A, B]=[0, L]$, the optimization problem can be written as

$$
\text { minimize: } \quad J(u)=\int \varphi(t) u(t, 0) d t+\int \psi(t) u(t, L) d t
$$

over all solutions whose initial data $\bar{u}$ satisfy the constraints (1.7)-(1.8).

Under natural assumptions on the cost functions $\varphi, \psi$, we will show that, for every given $\kappa>0$, this optimization problem has a unique solution. The corresponding density $\rho=\rho(t, x)$ 

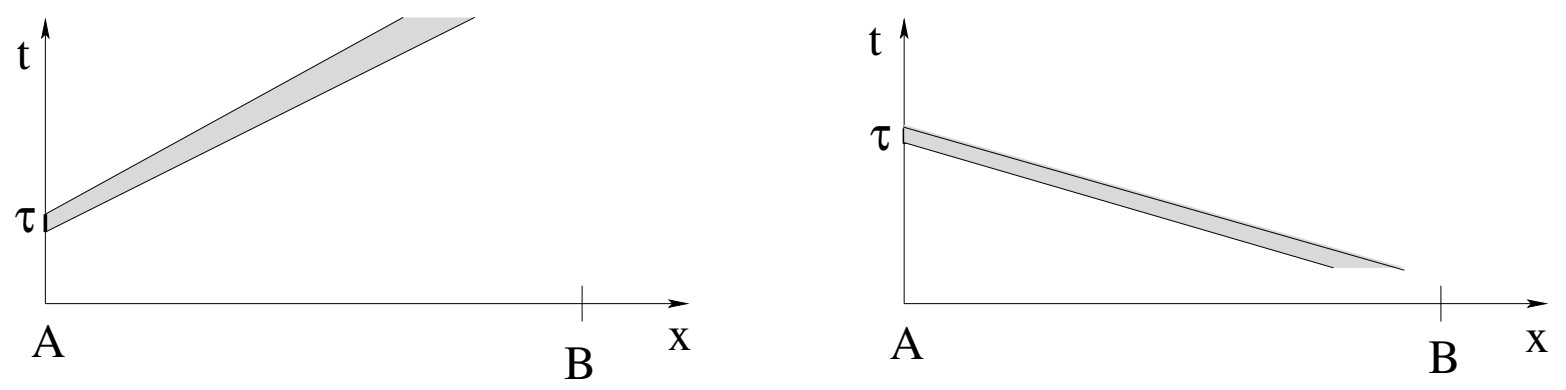

Figure 2: Left: when $\rho<\rho^{*}$, the characteristic speed is positive and characteristics starting from the boundary, where $x=A$, flow inside the domain. Right: when $\rho>\rho^{*}$, as time increases, characteristics exit from the domain.

in (1.3) admits a simple mathematical description. It has compact support and contains no shocks.

In absence of a higher authority who can force each driver to depart at a specific time, however, this solution is not likely to occur in practice. Indeed, it yields different costs to different drivers, depending on their starting time. In other words, the globally optimal solution is not a Nash equilibrium. As we shall define more precisely in Section 3, a Nash equilibrium is a solution where no driver can lower his individual cost by choosing a different departure time.

To construct a Nash solution, one needs to modify the model, allowing for arbitrarily large departure rates. We thus assume that, if drivers arrive at the beginning of the highway at a rate $\bar{u}(t)>M$ larger than the maximum flux defined at (1.7), they join a queue. The length of this queue $q(t) \geq 0$ and the flux of cars entering the highway are determined as follows.

- Either $q(t)=0$, or $\dot{q}(t) \doteq \frac{d}{d t} q(t)=\bar{u}(t)-M$.

- Instead of (1.11), the conservation law (1.10) is solved with initial condition

$$
u(t, 0+)=\rho(t, 0+) v(\rho(t, 0+))=\left\{\begin{array}{rll}
M & \text { if } q(t)>0 \\
\bar{u}(t) & \text { if } q(t)=0 .
\end{array}\right.
$$

Remarkably, it turns out that the Lax formula provides the correct solution also for this more general model, without requiring any modification. Indeed, let

$$
U(t, x) \doteq \int_{-\infty}^{t} u(\tau, x) d \tau
$$

be the total number of drivers that have crossed the point $x$ along the highway at some time $\leq t$. Let $Q(t)$ denote the number of drivers that have started their journey at a time $\leq t$ (joining the queue at the entrance of the highway, if there is any). Then the function $U$ provides a solution to the Cauchy problem

$$
U_{x}+f\left(U_{t}\right)=0, \quad U(t, 0)=\bar{U}(t) \doteq \inf \{Q(\tau)+M(t-\tau) ; \tau \leq t\} .
$$

Interpreting $U=U(t, x)$ as the value function for an auxiliary optimization problem, for every $x>0$ the solution of (1.13) is provided by

$$
U(t, x) \doteq \inf _{\tau}\left\{x f^{*}\left(\frac{t-\tau}{x}\right)+Q(\tau)\right\}
$$


where $f^{*}$ is the Legendre transform of $f$.

Our analysis shows that, for every $\kappa>0$, there exists a unique right-continuous, nondecreasing function $t \mapsto Q(t)$, with

$$
Q(-\infty)=0, \quad Q(+\infty)=\kappa,
$$

such that the solution of (1.13) yields a Nash equilibrium. This implies that all drivers face exactly the same cost, regardless of the time at which they decide to join the queue.

The remainder of the paper is organized as follows. Section 2 deals with an optimization problem for a scalar conservation law, where the cost depends only on the initial and terminal profile of the solution. Rather than (1.10), we write the conservation law in the more familiar form

$$
u_{t}+f(u)_{x}=0 \quad x \in \mathbb{R}, \quad t \in[0, T] .
$$

Of course, switching the variables $t$ and $x$ is purely for notational convenience. Under suitable assumptions on the cost functional, we prove the existence of a unique globally optimal solution $u=u(t, x)$. By deriving a set of necessary conditions for optimality, the optimal solution can be accurately described.

In Section 3 we state a precise concept of Nash equilibrium solution, writing the conservation law (1.16) in the integrated form

$$
U_{t}+f\left(U_{x}\right)=0 \quad U(0, x)=Q(x) .
$$

For each $\kappa>0$ we show that there exists a unique initial condition $Q$ satisfying (1.15) which yields a Nash equilibrium. Using the Lax formula, this solution can also be described in detail.

In Section 4 we show how the previous results apply to the original traffic problem. As an example, the globally optimal solution and the Nash equilibrium solution are explicitly computed, in the case where the cost functions are given by (1.2) and the flux function is determined by (1.5).

We observe that, by introducing an additional time-dependent cost $\phi(t)$ at the entrance of the highway (at a toll booth), one can easily transform the globally optimal solution into a Nash equilibrium. This provides a natural strategy for optimal toll pricing.

For a basic introduction to scalar conservation laws and the Lax formula we refer to the books of Evans [6] or Smoller [17]. An extension of the Lax formula to initial-boundary value problems was derived in [14]. Optimality conditions for weak solutions of hyperbolic conservation laws, also in the presence of shocks, were obtained in $[3,4,18,19]$. However, since the present problem requires only an optimal choice of the initial data for a scalar conservation law, our derivation of necessary conditions will be self-contained, relying on a direct application of the Lax formula.

Various optimization problems for traffic flow, based on the Lighthill-Whitham conservation law model, have been considered in $[9,10,11,12]$. Global optima and equilibria for a different kind of transport problem were recently studied in [5], also providing results on the asymptotic stability of Nash equilibria. For an introduction to differential games and for recent applications to traffic flow on networks we refer to $[2,7]$ and [8], respectively. 


\section{Optimal solutions to a conservation law}

Consider the scalar conservation law

$$
u_{t}+f(u)_{x}=0 .
$$

Given a time $T>0$ and a constant $\kappa>0$, consider the following optimization problem. Among all the initial data

$$
u(0, x)=\bar{u}(x)
$$

satisfying the constraints

$$
0 \leq \bar{u}(x) \leq M, \quad \int \bar{u}(x) d x=\kappa,
$$

find one which minimizes the cost functional

$$
J(\bar{u}) \doteq \int \varphi(x) \bar{u}(x) d x+\int \psi(x) u(T, x) d x .
$$

Here $u(T, \cdot)$ is the value at time $t=T$ of the unique entropy admissible solution to the Cauchy problem (2.1)-(2.2).

In order to prove the existence of optimal solution and derive a set of necessary conditions for optimality, the following assumptions will be used.

(A1) The flux function $f:[0, M] \mapsto \mathbb{R}$ is continuous, increasing, and strictly convex. It is twice continuously differentiable on the open interval $] 0, M[$ and satisfies

$$
f(0)=0, \quad f^{\prime \prime}(u) \geq b>0 \quad \text { for } 0<u<M .
$$

(A2) The cost functions $\varphi, \psi$ are locally Lipschitz continuous and satisfy

$$
\varphi^{\prime}<0, \quad \psi, \psi^{\prime} \geq 0, \quad \lim _{x \rightarrow-\infty} \varphi(x)=+\infty, \quad \lim _{x \rightarrow+\infty}(\varphi(x)+\psi(x))=+\infty .
$$

By the assumption (A1) the map $u \mapsto f^{\prime}(u)$ is strictly increasing on the open interval $] 0, M[$. Hence it admits one-sided limits $f^{\prime}(0+), f^{\prime}(M-)$, possibly with $f^{\prime}(M-)=+\infty$. The inverse function $g \doteq\left(f^{\prime}\right)^{-1}$ is thus well defined on the closed (possibly unbounded) interval

$$
I \doteq\left[f^{\prime}(0+), f^{\prime}(M-)\right] .
$$

In other words, for each $\lambda \in I$ we define

$$
g(\lambda)=u \quad \text { if and only if } \quad f^{\prime}(u)=\lambda .
$$

Notice that $I$ is the set of all possible characteristic speeds, as $u$ ranges in $[0, M]$. It is convenient to extend $f$ to a function $f: \mathbb{R} \mapsto \mathbb{R} \cup\{+\infty\}$, by setting

$$
f(u) \doteq\left\{\begin{array}{cl}
f^{\prime}(0+) u & \text { if } u<0, \\
f(M)+f^{\prime}(M-)(u-M) & \text { if } u>M .
\end{array}\right.
$$


In the case where $f^{\prime}(M-)=+\infty$, it is understood that $f(u)=+\infty$ whenever $u>M$. Let

$$
f^{*}(p) \doteq \max _{u}\{p u-f(u)\}
$$

be the Legendre transform of $f$. Notice that $f^{*}(p)=+\infty$ if $p<f^{\prime}(0+)$ or $p>f^{\prime}(M-)$. Calling $u=u(p)$ the point where the maximum in (2.10) is attained, we have

$$
\frac{d}{d p} f^{*}(p)=u(p) \in[0, M[
$$

The unique entropy-admissible solution to the Cauchy problem (2.1)-(2.2) can now be obtained by the Lax formulas

$$
\begin{gathered}
y(t, x)=\operatorname{argmin}\left\{t f^{*}\left(\frac{x-y}{t}\right)+\int_{-\infty}^{y} \bar{u}(s) d s ; \frac{x-y}{t} \in I\right\}, \\
u(t, x)=g\left(\frac{x-y(t, x)}{t}\right) .
\end{gathered}
$$

One can show that, for all except countably many points $x \in \mathbb{R}$, the expression on the right hand side of (2.12) attains its global minimum at a single point $y(t, x)$. In case the minimum is attained at several points, we denote by $y^{-}(t, x)$ and $y^{+}(t, x)$ respectively the smallest and the largest of such points. One has

$$
y^{+}\left(t, x_{1}\right) \leq y^{-}\left(t, x_{2}\right) \quad \text { whenever } \quad x_{1}<x_{2} .
$$

Moreover, under the assumption $f^{\prime \prime}(u) \geq b$, the Oleinik estimates hold:

$$
u\left(t, x_{2}\right)-u\left(t, x_{2}\right) \leq \frac{x_{2}-x_{1}}{b t} \quad \text { for all } t>0, x_{1}<x_{2} .
$$

Lemma 1. Let the flux function $f$ satisfy the assumptions (A1). Let $u=u(t, x)$ be a solution whose initial condition $u(0, x)=\bar{u}(x)$ satisfies (2.3). Then for any given $T>0$ the following holds.

(i) There exists $R$ large enough such that

$$
x-R \leq y(T, x) \leq x \quad \text { for all } x \in \mathbb{R} .
$$

(ii) Given any $\varepsilon>0$, there exists $\delta>0$ such that, for every solution $\tilde{u}$ whose initial data satisfy $\|u(0, \cdot)-\tilde{u}(0, \cdot)\|_{\mathbf{L}^{1}} \leq \delta$, one has

$$
\left[\tilde{y}^{-}(T, x), \tilde{y}^{+}(T, x)\right] \subseteq\left[y^{-}(T, x-\varepsilon)-\varepsilon, y^{+}(T, x+\varepsilon)+\varepsilon\right] \quad \text { for all } x \in \mathbb{R} .
$$

Here $\tilde{y}^{ \pm}$is defined in the same way as $y^{ \pm}$, replacing $u$ with $\tilde{u}$.

Proof. (i) By construction, $\frac{x-y(T, x)}{T} \in I \subseteq[0, \infty[$. 
If the set of characteristic speeds $I$ is bounded, it suffices to take $R \doteq T \cdot f^{\prime}(M)$. Notice that in this case (2.15) remains valid even without the assumption $\int u(0, x) d x=\kappa$.

Next, assume that $f^{\prime}(u) \rightarrow+\infty$ as $u \rightarrow M-$. Observing that $f^{*}(p) \geq p M-f(M)$, we have the estimate

$$
\Phi(y) \doteq T f^{*}\left(\frac{x-y}{T}\right)-\int_{-\infty}^{y} \bar{u}(s) d s \geq T\left(\frac{x-y}{T} M-f(M)\right)-\int_{-\infty}^{y} \bar{u}(s) d s
$$

Hence

as soon as

$$
\Phi(y)-\Phi(x) \geq(x-y) M-T f(M)-\int_{y}^{x} \bar{u}(s) d s>0
$$

$$
x-y>R \doteq \frac{T f(M)+\kappa}{M} .
$$

Hence the minimum in (2.12) can be attained at the point $y$ only if (2.15) holds.

(ii) Assuming that the conclusion does not hold, we shall derive a contradiction. Let $\bar{u}_{n}: \mathbb{R} \mapsto$ $[0, M]$ be a sequence of initial data with $\left\|\bar{u}_{n}-\bar{u}\right\|_{\mathbf{L}^{1}} \rightarrow 0$. Assume that there exists a sequence of points $x_{n}$ such that

$$
y_{n}^{+}\left(T, x_{n}\right)>y^{+}\left(T, x_{n}+\varepsilon\right)+\varepsilon
$$

for every $n \geq 1$. Here $y_{n}^{+}$refers to the initial data $\bar{u}_{n}$, while $y^{+}$refers to the initial data $\bar{u}$. (The case where $y_{n}^{-}\left(T, x_{n}\right)<y^{+}\left(T, x_{n}+\varepsilon\right)-\varepsilon$ is entirely similar.)

By the optimality of $y^{+}\left(T, x_{n}+\varepsilon\right)$ and $y_{n}^{+}\left(T, x_{n}\right)$, we obtain

$$
\begin{aligned}
& T f^{*}\left(\frac{x_{n}+\varepsilon-y^{+}\left(T, x_{n}+\varepsilon\right)}{T}\right)+\int_{-\infty}^{y^{+}\left(T, x_{n}+\varepsilon\right)} \bar{u}(s) d s \leq T f^{*}\left(\frac{x_{n}+\varepsilon-y_{n}^{+}\left(T, x_{n}\right)}{T}\right)+\int_{-\infty}^{y_{n}^{+}\left(T, x_{n}\right)} \bar{u}(s) d s, \\
& T f^{*}\left(\frac{x_{n}-y_{n}^{+}\left(T, x_{n}\right)}{T}\right)+\int_{-\infty}^{y_{n}^{+}\left(T, x_{n}\right)} \bar{u}_{n}(s) d s \leq T f^{*}\left(\frac{x_{n}-y^{+}\left(T, x_{n}+\varepsilon\right)}{T}\right)+\int_{-\infty}^{y^{+}\left(T, x_{n}+\varepsilon\right)} \bar{u}_{n}(s) d s .
\end{aligned}
$$

For notational convenience, from now on we shall write

$$
y_{n, \varepsilon}^{+} \doteq y^{+}\left(T, x_{n}+\varepsilon\right), \quad y_{n}^{+} \doteq y_{n}^{+}\left(T, x_{n}\right) .
$$

The above inequalities imply

$$
\begin{aligned}
T f^{*} & \left(\frac{x_{n}+\varepsilon-y_{n, \varepsilon}^{+}}{T}\right)-T f^{*}\left(\frac{x_{n}-y_{n, \varepsilon}^{+}}{T}\right)+T f^{*}\left(\frac{x_{n}-y_{n}^{+}}{T}\right)-T f^{*}\left(\frac{x_{n}+\varepsilon-y_{n}^{+}}{T}\right) \\
& \leq \int_{y_{n, \varepsilon}}^{y_{n}^{+}}\left(\bar{u}(s)-\bar{u}_{n}(s)\right) d s .
\end{aligned}
$$

By the assumption (A1), the flux function $f$ is increasing and strictly convex on $[0, M], \mathcal{C}^{2}$ on ] $0, M\left[\right.$. Hence $f^{*}$ is differentiable and its derivative $\left(f^{*}\right)^{\prime}=\left(f^{\prime}\right)^{-1}$ is also strictly increasing. Introducing the notations $z_{n}=\left(x_{n}-y_{n}^{+}\right) / T, z_{n, \varepsilon}=\left(x_{n}-y_{n, \varepsilon}^{+}\right) / T$, the inequality (2.18) can be written as

$$
T \int_{z_{n, \varepsilon}}^{z_{n, \varepsilon}+\varepsilon / T}\left(f^{*}\right)^{\prime}(s) d s-T \int_{z_{n}}^{z_{n}+\varepsilon / T}\left(f^{*}\right)^{\prime}(s) d s \leq \int_{y_{n, \varepsilon}}^{y_{n}^{+}}\left(\bar{u}(s)-\bar{u}_{n}(s)\right) d s .
$$


The fact that $\left(f^{*}\right)^{\prime}$ is strictly increasing implies that

$$
\int_{a}^{a+\varepsilon / T}\left(f^{*}\right)^{\prime}(s) d s-\int_{b}^{b+\varepsilon / T}\left(f^{*}\right)^{\prime}(s) d s \geq \delta,
$$

for some constant $\delta \geq 0$ and all $a, b \in\left[f^{\prime}(0+), R / T\right]$ with $b \geq a+\varepsilon$.

By (2.17), for every $n \geq 1$ we have $z_{n}+\varepsilon / T \leq z_{n, \varepsilon}$. Hence the left hand side of (2.19) remains $\geq \delta$ for every $n$, while the right hand side goes to zero. This achieves a contradiction.

\subsection{Existence of an optimal solution.}

The goal of this section is to prove the existence of an optimal solution to the minimization problem described at (2.1)-(2.4). As a preliminary, we show that under the assumptions (A1)-(A2) the initial data $\bar{u}$ which provides the optimum should have bounded support.

Lemma 2. Let the assumptions (A1)-(A2) hold. Then there exists a constant $R_{2}>0$ with the following property. If $\bar{u}(x)$ is any initial data satisfying (2.3), then there exists a second initial data $\bar{u}^{\dagger}$ satisfying (2.3), supported inside the interval $\left[-R_{2}, R_{2}\right]$, and such that the corresponding costs in (2.4) satisfy

$$
J\left(\bar{u}^{\dagger}\right) \leq J(\bar{u})
$$

The above inequality is strict, unless $\bar{u}$ already vanishes outside $\left[-R_{2}, R_{2}\right]$.

Proof. Choose a radius $R_{1}>0$ such that $2 M R_{1}=\kappa$. Let $R$ be the constant introduced at (2.15) and choose a radius $R_{2}>R_{1}+2 R$ large enough so that

$$
\max _{|x| \leq R_{1}} \varphi(x)+\max _{x \in\left[-R_{1}, R_{1}+R\right]} \psi(x)<\min _{|x| \geq R_{2}} \varphi(x)+\min _{|x| \geq R_{2}-R} \psi(x) .
$$

Such a radius certainly exists, because the left hand side of (2.21) is a fixed number, while by (2.6) the right hand side becomes arbitrarily large as $R_{2} \rightarrow+\infty$.

Now let $\bar{u}(\cdot)$ be an initial data satisfying (2.3). By the choice of $R_{1}$, there exists a function $\bar{u}^{\dagger}$ which also satisfies (2.3), and such that

$$
\begin{cases}\bar{u}^{\dagger}(x) \geq \bar{u}(x) & \text { if }|x| \leq R_{1}, \\ \bar{u}^{\dagger}(x)=\bar{u}(x) & \text { if } R_{1}<|x|<R_{2}, \\ \bar{u}^{\dagger}(x)=0 & \text { if }|x| \geq R_{2} .\end{cases}
$$

We claim that (2.20) holds. Indeed, observe that, by finite propagation speed, for all $t \in[0, T]$ we have

$$
\begin{aligned}
& \begin{cases}u^{\dagger}(t, x) \geq u(t, x) & \text { if }|x| \leq R_{1}+R \\
u^{\dagger}(t, x) \leq u(t, x) & \text { if }|x| \geq R_{2}-R\end{cases} \\
& \int_{|x| \leq R_{1}+R}\left(u^{\dagger}(t, x)-u(t, x)\right) d x=\int_{|x| \geq R_{2}-R}\left(u(t, x)-u^{\dagger}(t, x)\right) d x=\int_{|x| \geq R_{2}} \bar{u}(x) d x .
\end{aligned}
$$


Recalling (2.21), from (2.23)-(2.24) we thus obtain

$$
\begin{aligned}
J\left(\bar{u}^{\dagger}\right)-J(\bar{u})= & \int_{|x| \leq R_{1}} \varphi(x)\left(\bar{u}^{\dagger}(x)-\bar{u}(x)\right) d x+\int_{|x| \leq R_{1}+R} \psi(x)\left(u^{\dagger}(T, x)-u(T, x)\right) d x \\
& \quad-\int_{|x| \geq R_{2}} \varphi(x)\left(\bar{u}(x)-\bar{u}^{\dagger}(x)\right) d x-\int_{|x| \geq R_{2}-R} \psi(x)\left(u(T, x)-u^{\dagger}(T, x)\right) d x \\
\leq & \left(\max _{|x| \leq R_{1}} \varphi(x)+\max _{x \in\left[-R_{1}, R_{1}+R\right]} \psi(x)\right) \cdot \int_{|x| \geq R_{2}} \bar{u}(x) d x \\
& -\left(\min _{|x| \geq R_{2}} \varphi(x)+\min _{|x| \geq R_{2}-R} \psi(x)\right) \cdot \int_{|x| \geq R_{2}} \bar{u}(x) d x \\
\leq & 0 .
\end{aligned}
$$

Observe that the above inequality is strict, except in the case where $\bar{u}^{\dagger}=\bar{u}$.

Theorem 1. Let the assumptions (A1)-(A2) hold. Then, for any given $T, \kappa>0$, there exists an initial data $\bar{u}$ satisfying the constraint (2.3) and such that the corresponding entropy weak solution $u=u(t, x)$ to the conservation law (2.1) minimizes the cost functional (2.4).

Proof. Let $\left(\bar{u}_{n}\right)_{n \geq 1}$ be a minimizing sequence. Because of Lemma 2 , it is not restrictive to assume that all functions $\bar{u}_{n}$ vanish outside the interval $\left[-R_{2}, R_{2}\right]$. By taking a subsequence we can assume the weak convergence $\bar{u}_{n} \rightarrow \bar{u}$ for some $u \in \mathbf{L}^{1}$. Since all functions $\bar{u}_{n}$ satisfy the constraints (2.3), we clearly have

$$
\bar{u}(x) \in[0, M], \quad \int \bar{u}(s) d s=\kappa, \quad \bar{u}(x)=0 \quad \text { for }|x|>R_{2},
$$

Because of the Oleinik type estimates (2.14), for every $t>0$ the functions $u_{n}(t, \cdot)$ have uniformly bounded variation. In turn, this implies that the maps $t \mapsto u_{n}(t, \cdot)$ are uniformly Lipschitz continuous from $[\delta, T]$ into $\mathbf{L}^{1}(\mathbb{R})$, for any fixed $\delta>0$. Using a version of Helly's compacness theorem (see for example Theorem 2.4 in [1]), by taking a further subsequence we can achieve the convergence

$$
\left\|u_{n}(t, \cdot)-u(t, \cdot)\right\|_{\mathbf{L}^{1}(\mathbb{R})} \rightarrow 0 \quad \text { for all } 0<t \leq T,
$$

for some limit function $u=u(t, x)$. Here the map $t \mapsto u(t, \cdot) \in \mathbf{L}^{1}(\mathbb{R})$ is Lipschitz continuous restricted to any subinterval $[\delta, T]$, with $\delta>0$. The fact that $u$ is the unique entropy weak solution to the Cauchy problem (2.1)-(2.2) can be proved by checking that the Lax identity (2.12)-(2.13) is satisfied. This is clear, because the weak convergence of the initial data $\bar{u}_{n} \rightarrow \bar{u}$ implies the uniform convergence of the integral functions

$$
\bar{U}_{n}(x) \doteq \int_{-\infty}^{x} \bar{u}_{n}(s) d s \rightarrow \bar{U}(x) \doteq \int_{-\infty}^{x} \bar{u}(s) d s .
$$

Moreover, (2.25) yields the convergence $U_{n}(t, x) \rightarrow U(t, x)$, uniformly in $x$, for every fixed $t>0$.

The weak convergence $\bar{u}_{n} \rightarrow \bar{u}$, together with the strong convergence $\left\|u_{n}(T, \cdot)-u(T, \cdot)\right\|_{\mathbf{L}^{1}(\mathbb{R})} \rightarrow 0$ and the uniform boundedness of the supports, yield

$$
\lim _{n \rightarrow \infty}\left(\int \varphi(x) \bar{u}_{n}(x) d x+\int \psi(x) u_{n}(T, x) d x\right)=\int \varphi(x) \bar{u}(x) d x+\int \psi(x) u(T, x) d x .
$$


Hence $u$ is an optimal solution.

\subsection{Necessary conditions for optimality.}

Let $u=u(t, x)$ be an optimal solution of the conservation law (2.1), providing a minimum to the functional (2.4), subject to the constraints (2.3) on the initial data. Aim of this section is to provide a detailed description of $u$, deriving a set of necessary conditions for optimality.

For each $y \in \mathbb{R}$, consider the maximal and minimal backward characteristics through $(T, y)$. Recall that, by (A1), the characteristic speed $f^{\prime}(u)$ is a strictly increasing function of $u$. Hence $u(T, \cdot)$ has locally bounded variation, satisfies a one-sided Lipschitz condition, and has at most countably many downward jumps. Calling $u(T, x+) \leq u(T, x-)$ the right and left limits of $u(T, \cdot)$ at the point $x$, we then define

$$
\begin{aligned}
& y^{-}(x) \doteq x-T f^{\prime}(u(T, x-)), \\
& y^{+}(x) \doteq x-T f^{\prime}(u(T, x+)),
\end{aligned}
$$

Notice that $y^{-}, y^{+}$are the initial points of the minimal and maximal backward characteristics through the point $(T, x)$. We recall that $y$ is called a Lebesgue point for the function $\bar{u}$ if

$$
\lim _{\rho \rightarrow 0+} \frac{1}{\rho} \int_{y-\rho}^{y+\rho}|\bar{u}(s)-\bar{u}(y)| d s=0 .
$$

Since $\bar{u} \in \mathbf{L}^{1}(\mathbb{R})$, the above limit holds at a.e. $y \in \mathbb{R}$.

Remark 2. If $y$ is a Lebesgue point for $\bar{u}$, then $y \in I(x)$ for a unique point $x$. In other words, $y$ cannot be the center of a rarefaction wave. Indeed, assume, on the contrary, that $y \in I\left(x_{1}\right) \cap I\left(x_{2}\right)$ for some $x_{1}<x_{2}$ Then

$$
y=\operatorname{argmin}\left\{T f^{*}\left(\frac{x-y}{T}\right)+\int_{-\infty}^{y} \bar{u}(s) d s\right\} \quad \text { for all } x \in\left[x_{1}, x_{2}\right] .
$$

For every $\rho>0$ small, this implies:

$$
\begin{gathered}
\int_{y}^{y+\rho} \bar{u}(s) d s \geq T f^{*}\left(\frac{x_{2}-y}{T}\right)-T f^{*}\left(\frac{x_{2}-(y+\rho)}{T}\right)=T\left(f^{*}\right)^{\prime}\left(\xi_{2}\right) \cdot \frac{\rho}{T}, \\
\int_{y-\rho}^{y} \bar{u}(s) d s \leq T f^{*}\left(\frac{x_{2}-(y-\rho)}{T}\right)-T f^{*}\left(\frac{x_{2}-y}{T}\right)=T\left(f^{*}\right)^{\prime}\left(\xi_{1}\right) \cdot \frac{\rho}{T}, \\
\quad \text { for some } \xi_{1} \in\left[\frac{x_{1}-y}{T}, \frac{x_{1}-(y-\rho)}{T}\right], \quad \xi_{2} \in\left[\frac{x_{2}-(y+\rho)}{T}, \frac{x_{2}-y}{T}\right] .
\end{gathered}
$$

Then

$$
\begin{aligned}
\frac{1}{\rho} \int_{y-\rho}^{y+\rho}|\bar{u}(s)-\bar{u}(y)| d s & \geq \frac{1}{\rho} \int_{y}^{y+\rho} \bar{u}(s)-\bar{u}(y) d s-\frac{1}{\rho} \int_{y-\rho}^{y} \bar{u}(s)-\bar{u}(y) d s \\
& \geq\left(f^{*}\right)^{\prime}\left(\xi_{2}\right)-\left(f^{*}\right)^{\prime}\left(\xi_{1}\right) \geq\left(f^{*}\right)^{\prime}\left(\frac{x_{1}-y}{T}\right)-\left(f^{*}\right)^{\prime}\left(\frac{x_{2}-y}{T}\right),
\end{aligned}
$$


which is impossible if $y$ is a Lebesgue point.

We now derive a necessary condition valid at all Lebesgue points of $\bar{u}$.

Lemma 3. Let $u=u(t, x)$ be an optimal solution of (2.1), with initial data $u(0, \cdot)=\bar{u}$ satisfying the constraints (2.3), and providing a minimum to the functional (2.4). Assume that the flux function $f$ satisfies the assumptions (A1) and that the cost functions $\varphi, \psi$ are continuous.

If $y_{1}, y_{2}$ are Lebesgue points of $\bar{u}=u(0, \cdot)$, with

$$
\left\{\begin{array} { l } 
{ y _ { 1 } \in I ( x _ { 1 } ) , } \\
{ y _ { 2 } \in I ( x _ { 2 } ) , }
\end{array} \quad \left\{\begin{array}{l}
\bar{u}\left(y_{1}\right)>0 \\
\bar{u}\left(y_{2}\right)<M
\end{array}\right.\right.
$$

then

$$
\varphi\left(y_{1}\right)+\psi\left(x_{1}\right) \leq \varphi\left(y_{2}\right)+\psi\left(x_{2}\right)
$$

Proof. Since $y_{1}, y_{2}$ are Lebesgue points of $\bar{u}$, by Remark 2, the points $x_{1}<x_{2}$ in (2.27) are uniquely determined. Assuming that (2.28) fails, we will derive a contradiction. Indeed, we will construct a new initial data $\bar{u}^{\dagger}$ which is slightly smaller than $\bar{u}$ in a neighborhood of $y_{1}$ and slightly larger that $\bar{u}$ in a neighborhood of $y_{2}$, thus yielding a lower total cost.

1. Choose $\delta>0$ such that

$$
\max _{\left|y-y_{1}\right| \leq \delta} \varphi(y)+\max _{\left|x-x_{1}\right| \leq \delta} \psi(x)<\min _{\left|y-y_{2}\right| \leq \delta} \varphi(y)+\min _{\left|x-x_{2}\right| \leq \delta} \psi(x)
$$

2. Choose points $x_{1}^{ \pm}, x_{2}^{ \pm}$where $\bar{u}$ is continuous and such that

$$
x_{1}-\delta<x_{1}^{-}<x_{1}<x_{1}^{+}<x_{1}+\delta, \quad x_{2}-\delta<x_{2}^{-}<x_{2}<x_{2}^{+}<x_{2}+\delta .
$$

Notice that the continuity of $\bar{u}$ at $x_{i}^{ \pm}$implies that the corresponding points

$$
y\left(x_{1}^{-}\right)<y_{1}<y\left(x_{1}^{+}\right), \quad y\left(x_{2}^{-}\right)<y_{2}<y\left(x_{2}^{+}\right)
$$

are uniquely defined. Consider the points

$$
\left\{\begin{array} { l } 
{ y _ { 1 } ^ { - } \doteq \operatorname { m a x } \{ y ( x _ { 1 } ^ { - } ) , y _ { 1 } - \delta \} < y _ { 1 } , } \\
{ y _ { 1 } ^ { + } \doteq \operatorname { m i n } \{ y ( x _ { 1 } ^ { + } ) , y _ { 1 } + \delta \} > y _ { 1 } , }
\end{array} \quad \left\{\begin{array}{l}
y_{2}^{-} \doteq \max \left\{y\left(x_{2}^{-}\right), y_{2}-\delta\right\}<y_{2}, \\
y_{2}^{+} \doteq \min \left\{y\left(x_{2}^{+}\right), y_{2}+\delta\right\}>y_{2} .
\end{array}\right.\right.
$$

3. By the assumption (2.27), for every $\varepsilon>0$ sufficiently small we can construct a second initial condition $\bar{u}^{\dagger}$ such that

$$
\begin{cases}\bar{u}^{\dagger}(y)=\bar{u}(y) & \text { if } y \notin\left[y_{1}^{-}, y_{1}^{+}\right] \cup\left[y_{2}^{-}, y_{2}^{+}\right], \\ \bar{u}^{\dagger}(y) \leq \bar{u}(y) & \text { if } y \in\left[y_{1}^{-}, y_{1}^{+}\right] \\ \bar{u}^{\dagger}(y) \geq \bar{u}(y) & \text { if } y \in\left[y_{2}^{-}, y_{2}^{+}\right]\end{cases}
$$




$$
\int_{y_{1}^{-}}^{y_{1}^{+}}\left[\bar{u}(s)-\bar{u}^{\dagger}(s)\right] d s=\int_{y_{2}^{-}}^{y_{2}^{+}}\left[\bar{u}^{\dagger}(s)-\bar{u}(s)\right] d s=\varepsilon .
$$

4. By (2.30), using part (ii) of Lemma 1 we can choose $\varepsilon>0$ sufficiently small such that the corresponding solutions $u, u^{\dagger}$ of (2.1) satisfy

$$
\begin{aligned}
& \begin{cases}u^{\dagger}(T, x)=u(T, x) & \text { for all } x \notin\left[x_{1}-\delta, x_{1}+\delta\right] \cup\left[x_{2}-\delta, x_{2}+\delta\right], \\
u^{\dagger}(T, x) \leq u(T, x) & \text { for all } x \in\left[x_{1}-\delta, x_{1}+\delta\right], \\
u^{\dagger}(T, x) \geq u(T, x) & \text { for all } x \in\left[x_{2}-\delta, x_{2}+\delta\right] .\end{cases} \\
& \int_{x_{1}-\delta}^{x_{1}+\delta}\left[u(T, x)-u^{\dagger}(T, x)\right] d x=\int_{x_{2}-\delta}^{x_{2}+\delta}\left[u^{\dagger}(T, x)-u(T, x)\right] d x=\varepsilon .
\end{aligned}
$$

By the strict inequality (2.29), the above relations imply that $J\left(\bar{u}^{\dagger}\right)<J(\bar{u})$, contradicting the optimality of $\bar{u}$.

The following theorem yields a precise description of the optimal solution.

Theorem 2. In addition to (A1)-(A2), assume that $f^{\prime}(u) \rightarrow+\infty$ as $u \rightarrow M-$. Then, for every $\kappa>0$, the optimization problem described at (2.1)-(2.4) has a unique solution $u=u(t, x)$. In addition, one has:

(I) No shocks are present, hence $u$ is continuous for $t>0$. Moreover,

$$
\sup _{t \in[0, T], x \in \mathbb{R}} u(t, x)<M .
$$

(II) For some constant $c=c(\kappa)$, this optimal solution admits the following characterization.

For every $x \in \mathbb{R}$, let $y_{c}(x)$ be the unique point such that

$$
\varphi\left(y_{c}(x)\right)+\psi(x)=c .
$$

Then, along the segment with endpoints $\left(0, y_{c}(x)\right),(T, x)$, the function $u$ is defined according to:

(i) If $\frac{x-y_{c}(x)}{T}=f^{\prime}(v)$ for some $v>0$, then

$$
\left.\left.u\left(t, \frac{t}{T} x+\frac{T-t}{T} y_{c}(x)\right)=v \quad \text { for all } t \in\right] 0, T\right]
$$

(ii) If $\frac{x-y_{c}(x)}{T} \leq f^{\prime}(0+)$, then

$$
\left.\left.u\left(t, \frac{t}{T} x+\frac{T-t}{T} y_{c}(x)\right)=0 \quad \text { for all } t \in\right] 0, T\right] .
$$


Proof. 1. Assume that the optimal solution contains a shock. Call $\bar{x}$ the position of this shock at time $t=T$, and let

$$
u^{-} \doteq u(T, \bar{x}-)>u^{+} \doteq u(T, \bar{x}+)
$$

be the left and right limits of $u(T, \cdot)$ at this point. Notice that these limits exists, because $u(T, \cdot)$ has bounded variation. Computing the minimal and maximal backward characteristics through the point $(T, \bar{x})$ we find

$$
y^{-} \doteq y^{-}(0, \bar{x})=\bar{x}-T f^{\prime}\left(u^{-}\right), \quad y^{+} \doteq y^{+}(0, \bar{x})=\bar{x}-T f^{\prime}\left(u^{+}\right) .
$$

Consider any two points $y_{1}, y_{2} \in\left[y^{-}, y^{+}\right]$, say with $y_{1}<y_{2}$. Since $\varphi\left(y_{1}\right)>\varphi\left(y_{2}\right)$, we must have either $\bar{u}\left(y_{1}\right)=0$ or $\bar{u}\left(y_{2}\right)=M$. In the opposite case Lemma 3 would yield a contradiction. We thus conclude that there exists a point $\xi \in\left[y^{-}, y^{+}\right]$such that

$$
\begin{cases}\bar{u}(y)=0 & \text { if } y^{-}<y<\xi \\ \bar{u}(y)=M & \text { if } \xi<y<y^{+}\end{cases}
$$

We claim that (2.35) also leads to a contradiction. Indeed, if $\xi>y^{-}$, then

$$
T f^{*}\left(\frac{x-\xi}{T}\right)+\int_{-\infty}^{\xi} \bar{u}(s) d s<T f^{*}\left(\frac{x-y^{-}}{T}\right)+\int_{-\infty}^{y^{-}} \bar{u}(s) d s .
$$

This contradicts the optimality of $y^{-}$in the Lax representation (2.12). On the other hand, if $\xi=y^{-}$, since both $y^{-}$and $y^{+}$yield the minimum in (2.12), we have

$$
\begin{aligned}
0 & =T f^{*}\left(\frac{\bar{x}-y^{-}}{T}\right)-\int_{-\infty}^{y^{-}} \bar{u}(s) d s-T f^{*}\left(\frac{\bar{x}-y^{+}}{T}\right)+\int_{-\infty}^{y^{+}} \bar{u}(s) d s \\
& =T \int_{\left(\bar{x}-y^{-}\right) / T}^{\left(\bar{x}-y^{+}\right) / T}\left(f^{*}\right)^{\prime}(p) d p+M\left(y^{+}-y^{-}\right) .
\end{aligned}
$$

Since $\left(f^{*}\right)^{\prime}(p)=u$ if and only if $f^{\prime}(u)=p$, the assumption $f^{\prime}(u) \rightarrow+\infty$ as $u \rightarrow M-$ implies that $\left(f^{*}\right)^{\prime}(p)<M$ for all $p \geq f^{\prime}(0+)$. Hence the right hand side of (2.36) is strictly negative and equality cannot hold. This contradiction shows that the optimal solution cannot contain shocks.

2. To prove (2.31), observe that by Lemma 2 the solution $u$ has compact support. To fix the ideas, let $\bar{u}=u(0, \cdot)$ be supported inside $\left[-R_{1}, R_{1}\right]$ and let $u(T, \cdot)$ be supported inside $\left[-R_{2}, R_{2}\right]$. Since $\lim _{u \rightarrow M-} f^{\prime}(u)=+\infty$, we can choose a value $h<M$ such that $f^{\prime}(h)>\left(R_{1}+R_{2}\right) / T$.

We claim that $\bar{u}(x)<h$ for all $x$. Otherwise, choose a point $x_{0}$ such that $\bar{u}\left(x_{0}\right) \geq h$. Then, since no shocks are present, computing the solution by the method of characteristics we find

$$
u\left(t, x_{0}+t f^{\prime}\left(\bar{u}\left(x_{0}\right)\right)\right)=\bar{u}\left(x_{0}\right) .
$$

Consider the point $x_{T} \doteq x_{0}+T f^{\prime}\left(\bar{u}\left(x_{0}\right)\right)$. Since $x_{0} \geq-R_{1}$, the choice of $h$ implies $x_{T}>R_{2}$. We thus have $u\left(T, x_{T}\right)=\bar{u}\left(x_{0}\right) \geq h>0$, contradicting the fact that the support of $u(T, \cdot)$ should be contained in $\left[-R_{2}, R_{2}\right]$. This proves our claim, and hence (2.31). 
3. Since no shocks are present, the solution $u$ is continuous for $t>0$. As a consequence, backward characteristics are uniquely defined. In particular, at $t=T$, for each $x \in \mathbb{R}$ the minimizer $y=y(T, x)$ in the Lax representation (2.12) is unique. We recall that $y(T, x)$ is the initial point on the characteristic line which ends at $(T, x)$.

4. By Lemma 3 and (2.31), there exists a unique constant $c$ such that the following holds. If $y=y(T, x)$ is Lebesgue point for the initial data $\bar{u}$, with $\bar{u}(y)>0$, then

$$
\varphi(y)+\psi(x)=c .
$$

Consider the map $x \mapsto y_{c}(x)$ implicitly defined by (4.1). Since the function $\varphi$ is strictly decreasing, the value $y_{c}(x)$ is well defined provided that $\varphi(+\infty)+\psi(x)<c$. For notational convenience, we set $y_{c}(x) \doteq+\infty$ if $\varphi(+\infty)+\psi(x) \geq c$. From the assumption $\psi^{\prime} \geq 0$ it follows that the map $x \mapsto y_{c}(x)$ is non-decreasing.

5. Assume that $y_{c}(x)$ is a Lebesgue point of $\bar{u}$ and $\bar{u}\left(y_{c}(x)\right)>0$. Then (4.1) and (2.37) together yield

$$
\varphi\left(y_{c}(x)\right)+\psi(x)=c=\varphi(y(T, x))+\psi(x) .
$$

Since $\varphi$ is strictly decreasing, this yields

$$
y_{c}(x)=y(T, x)=x-T f^{\prime}(u(T, x)) \text {. }
$$

Therefore, along the (characteristic) segment with endpoints $\left(0, y_{c}(x)\right),(T, x)$ and slope $f^{\prime}(v)$, $v=\bar{u}\left(y_{c}\right)=u(T, x)$, the function $u=u(t, x)$ is determined by $(2.33)$.

6. Observe that the second identity in (2.38) always holds, because the solution $u=u(t, x)$ is continuous for $t>0$ and backward characteristics are unique. In this step we prove that the first equality in (2.38) remains valid as long as $\frac{x-y_{c}(x)}{T}>f^{\prime}(0+)$, even if $y_{c}(x)$ is not a Lebesgue point of $\bar{u}$.

Indeed, if $y(T, x)<y_{c}(x)$, let $\left.\tilde{y} \in\right] y(T, x), y_{c}(x)[$ be a Lebesgue point of $\bar{u}$. Then

$$
\tilde{y}=y(T, \tilde{x}) \quad \text { for some } \quad \tilde{x} \doteq \tilde{y}+T f^{\prime}(\bar{u}(\tilde{y}))>x .
$$

Since

$$
\frac{\tilde{x}-\tilde{y}}{T}>\frac{x-y_{c}(x)}{T}>f^{\prime}(0+)
$$

it is clear that $\bar{u}(\tilde{y})>0$. By step 5 , we have $\tilde{y}=y(T, \tilde{x})=y_{c}(\tilde{x})$. Since $\tilde{x} \geq x$ but $\tilde{y}<y_{c}(x)$, recalling that $\varphi$ is strictly decreasing we obtain

$$
\varphi(y(T, \tilde{x}))+\psi(\tilde{x})=\varphi\left(y_{c}(\tilde{x})\right)+\varphi(y(T, \tilde{x}))<\varphi\left(y_{c}(x)\right)+\psi(x)
$$

contradicting the fact that all terms in the above expression are equal to $c$.

On the other hand, if $y(T, x)>y_{c}(x)$, let $\left.\tilde{y} \in\right] y_{c}(x), y(T, x)$ [ be a Lebesgue point of $\bar{u}$. Then

$$
\tilde{y}=y(T, \tilde{x}) \quad \text { for some } \quad \hat{x} \doteq \tilde{y}+T f^{\prime}(u(T, \tilde{y}))<x
$$


Since $u(0, \tilde{y})<M$, using Lemma 3 and the assumptions $\varphi^{\prime}<0, \psi^{\prime} \geq 0$ we obtain

$$
c \leq \varphi(\tilde{y})+\psi(\tilde{x})<\varphi\left(y_{c}(x)\right)+\psi(x),
$$

again reaching a contradiction.

We thus conclude that, if $\frac{x-y_{c}(x)}{T}=f^{\prime}(v)>f^{\prime}(0+)$, then $(2.33)$ holds.

7. Next, consider the case where $\frac{x-y_{c}(x)}{T} \leq f^{\prime}(0+)$. We claim that for all $\bar{x} \in\left[x, y_{c}(x)+\right.$ $\left.T f^{\prime}(0+)\right]$

$$
\frac{\bar{x}-y(T, \bar{x})}{T}=f^{\prime}(u(T, \bar{x}))=f^{\prime}(0+) .
$$

Indeed, if $f^{\prime}(u(T, \bar{x}))>f^{\prime}(0+)$, then

$$
y(T, \bar{x})=\bar{x}-T f^{\prime}(u(T, \bar{x}))<y_{c}(x) \quad \varphi(y(T, \bar{x}))+\psi(\bar{x})>c .
$$

As in the previous steps, we can then find a Lebesgue point $\tilde{y} \in] y(T, \bar{x}), y_{c}(x)[$ such that

$$
\varphi(\tilde{y})+\psi(\bar{x})>c, \quad \frac{\bar{x}-\tilde{y}}{T}>f^{\prime}(0+) .
$$

Then $\tilde{y}=y(T, \tilde{x})$, for some point $\tilde{x}$ satisfying

$$
\tilde{x}>\bar{x}, \quad \frac{\tilde{x}-\tilde{y}}{T}>\frac{\bar{x}-\tilde{y}}{T}>f^{\prime}(0+) .
$$

Hence $\bar{u}(\tilde{y})>0$. We thus have

$$
c=\varphi(\tilde{y})+\psi(\tilde{x})>\varphi\left(y_{c}(x)\right)+\psi(x),
$$

reaching contradiction to. This proves our claim.

Since $f^{\prime}$ is strictly increasing, from $(2.39)$ it follows that $u(T, \bar{x})=0$ for all $\bar{x} \in\left[x, y_{c}(x)+\right.$ $\left.T f^{\prime}(0+)\right]$, hence $(2.34)$ holds.

8. In this final step, we prove the uniqueness of the optimal solution.

Observe that, for each value of the constant $c \in \mathbb{R}$, the properties (2.33)-(2.34) yield a unique solution $u=u(t, x)$ of the conservation law (2.1). This solution is continuous for $t>0$, while the initial condition $u(0, \cdot)=\bar{u}^{(c)}(\cdot)$ contains at most countably many upward jumps (producing centered rarefaction waves).

Assume that, for some $\kappa>0$, there exists two optimal solutions, say $u_{1}, u_{2}$. If $u_{1} \neq u_{2}$, these solutions will satisfy (2.33)-(2.34) with different constants, say $c_{1}<c_{2}$. We show that this leads to a contradiction.

Since $\varphi$ is strictly decreasing, for every $x \in \mathbb{R}$ there exist unique values $y_{c_{2}}(x)<y_{c_{1}}(x)$ such that

$$
\varphi\left(y_{c_{1}}(x)\right)+\psi(x)=c_{1}, \quad \varphi\left(y_{c_{2}}(x)\right)+\psi(x)=c_{2} .
$$

For every $x$ such that $\frac{x-y_{c_{1}}(x)}{T}>f^{\prime}(0+)$, by the previous analysis the values of $u_{1}(T, x)$ and $u_{2}(T, x)$ are implicitly defined

$$
f^{\prime}\left(u_{1}(T, x)\right)=\frac{x-y_{c_{1}}(x)}{T}<\frac{x-y_{c_{2}}(x)}{T}=f^{\prime}\left(u_{2}(T, x)\right) .
$$


Since $f^{\prime}$ is strictly increasing, for every $x \in \mathbb{R}$ this provides the implication

$$
u_{1}(T, x)>0 \quad \Longrightarrow \quad u_{1}(T, x)<u_{2}(T, x) .
$$

Therefore, by conservation of the total mass we conclude

$$
\int u_{1}(0, x) d x=\int u_{1}(T, x) d x<\int u_{2}(T, x) d x=\int u_{2}(0, x) d x .
$$

This contradicts the assumption that all integrals are $=\kappa$, thus proving uniqueness.

Remark 3. The optimality conditions derived in Theorem 2 apply to traffic flow as well as to other models, such as supply-chains. All the analysis, based on the Lax formula, is valid for scalar conservation laws as long as the flux function is convex and depends only on the density of the conserved quantity.

On the other hand, this technique cannot be used when the flux depends also on the variables $t, x$, or in connection with second-order traffic models which are described by system of conservation laws. To analyze these more complex situations, necessary conditions for optimality can still be obtained in the form of a Pontryagin maximum principle $[3,4]$, but only within a class of piecewise regular solutions.

\section{The Nash equilibrium}

Aim of this section is to give a precise definition of Nash equilibrium solution for the above problem of traffic flow, and prove its existence and uniqueness.

Introducing the integral function

$$
U(t, x) \doteq \int_{-\infty}^{x} u(t, s) d s
$$

the conservation law (2.1) can be equivalently written as a Hamilton-Jacobi equation

$$
U_{t}+f\left(U_{x}\right)=0 .
$$

Throughout the following, $f$ denotes the flux function extended to the entire real line as in (2.9), while $f^{*}$ is the corresponding Legendre transform, defined at (2.10). For our application to traffic flow, one should keep in mind that the $x$ variable denotes time, while $t \in[0, T]$ denotes a point along the highway. Hence $U(t, x)$ measures the total number of cars that have crossed the point $t$ along the highway during the time interval ] $-\infty, x]$.

As initial data we shall consider any bounded non-decreasing function $Q: \mathbb{R} \mapsto \mathbb{R}^{+}$, with

$$
Q(-\infty)=0, \quad Q(+\infty)=\kappa
$$

Here $Q(x)$ denotes the total number of cars that have entered the queue at the entrance of the highway up to time $x$. Notice that $Q$ is continuous except for countably many times $x$. To fix the ideas, we shall consider the right-continuous version where $Q(x)=Q(x+)$ coincides with its right limit at every $x$. When needed, we shall denote by $Q(x-)=\lim _{y \rightarrow x-} Q(y)$ the left limit of $Q$ at $x$. 
For a given $Q(\cdot)$, consider the Lipschitz continuous function

$$
\bar{U}(x) \doteq \inf \{Q(y)+M(x-y) ; y \leq x\} \leq Q(x) .
$$

Notice that $Q(x)-\bar{U}(x)$ measures the length of the queue at time $x$, while $\bar{U}(x)$ denotes the total number of drivers that have actually departed (after clearing the queue) up to time $x$.

For $t>0$, the entropy-admissible solution to the Cauchy problem (3.1), (3.3) is provided by the Lax formula:

$U(t, x)=\min \left\{t f^{*}\left(\frac{x-y}{t}\right)+\bar{U}(y) ; y \in \mathbb{R}\right\}=\min \left\{t f^{*}\left(\frac{x-y}{t}\right)+Q(y-) ; y \in \mathbb{R}\right\}$.

Observe that the last two expressions in (3.4) are equal because $\left(f^{*}\right)^{\prime}(p) \leq M$ for all $p$, and $\left(f^{*}\right)^{\prime}(p) \rightarrow M$ as $p \rightarrow+\infty$. Moreover, $U(0+, x)=\bar{U}(x)$.

To visualize the profile of this solution at time $t=T$, it is convenient to introduce the function

$$
h(s) \doteq-T f^{*}\left(\frac{-s}{T}\right) .
$$

Observe that $h$ is a concave function. Setting $\mu \doteq T / f^{\prime}(0)$, one has

$$
h(s)=-\infty \text { for } s>-\mu, \quad h^{\prime}(-\mu)=0, \quad \lim _{s \rightarrow-\infty} h^{\prime}(s)=M .
$$

From (3.4) it now follows (see fig. 3)

$$
U(T, x)=\min _{y}\{\bar{U}(y)-h(y-x)\}=\min _{y}\{Q(y-)-h(y-x)\} .
$$

In other words, $U(T, x)$ is the amount by which we can shift upward the graph of $h(\cdot-x)$, before hitting the graph of $Q(\cdot)$ (or, equivalently, the graph of $\bar{U}$ ). From this construction it is clear that the map $x \mapsto U(T, x)$ is nondecreasing. For solutions with different initial data one has the comparison property

$$
Q(y) \leq \widetilde{Q}(y) \text { for all } y \in \mathbb{R} \quad \Longrightarrow \quad U(T, x) \leq \widetilde{U}(T, x) \text { for all } x \in \mathbb{R} .
$$

Given an initial data $Q(\cdot)$ as in $(3.2)$, for $\beta \in\left[0, \kappa\left[\right.\right.$ we define the points $x^{q}(\beta), x^{d}(\beta)$, and $x^{a}(\beta)$ by setting

$$
\left\{\begin{array}{l}
x^{q}(\beta)=\sup \{x \in \mathbb{R} ; \quad Q(x) \leq \beta\}, \\
x^{d}(\beta)=\sup \{x \in \mathbb{R} ; \quad U(0+, x) \leq \beta\}, \\
x^{a}(\beta)=\sup \{x \in \mathbb{R} ; \quad U(T, x) \leq \beta\} .
\end{array}\right.
$$

In the application to traffic flow, $\beta$ is a Lagrangian variable labeling a particular driver. In this case, $x^{q}(\beta)$ accounts for the time where this driver joins the queue, $x^{d}(\beta)$ is the actual departure time and $x^{a}(\beta)$ is the arrival time.

Remark 4. For all except countably many $\beta$, the points $x^{q}(\beta)$ and $x^{d}(\beta)$ are uniquely determined by the identities

$$
Q\left(x^{q}(\beta)-\right) \leq \beta \leq Q\left(x^{q}(\beta)\right), \quad \bar{U}\left(x^{d}(\beta)\right)=\beta .
$$




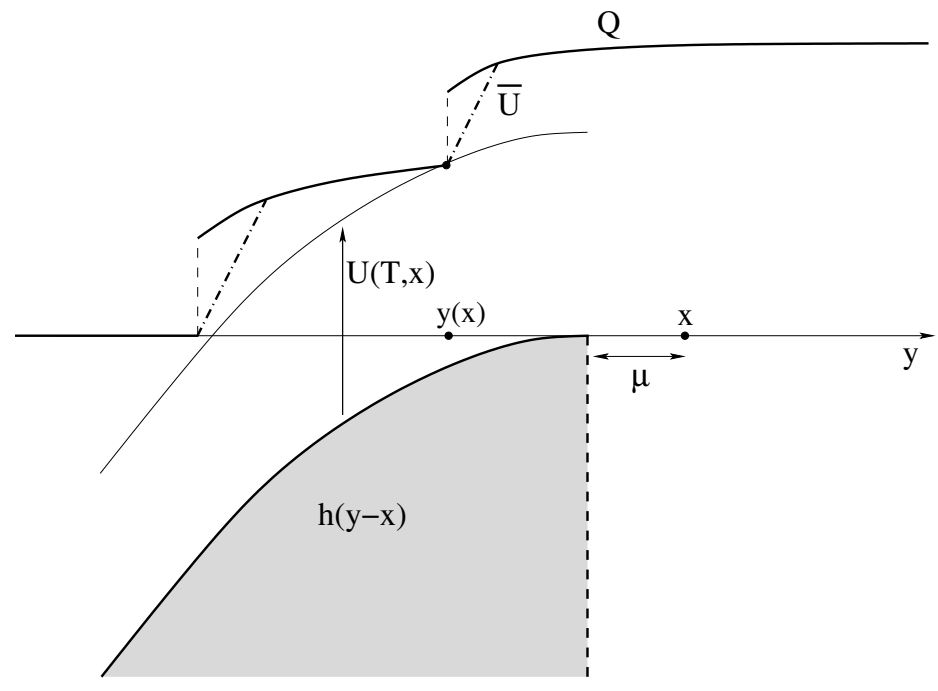

Figure 3: Constructing the profile $x \mapsto U(T, x)$ using the Lax formula. Since $h^{\prime} \leq M$, the supremum in (3.7) does not change if $Q(\cdot)$ is replaced by $\bar{U}(\cdot)$.

Moreover (see fig. 4), for a.e. $\beta$ the arrival time $x^{a}$ is determined as

$$
x^{a}(\beta)=\inf \{x ; Q(y) \geq \beta+h(y-x) \text { for all } y \leq x\} .
$$

More generally, for a driver that departs at time $x$, we define the arrival time as

$$
A(x) \doteq \max \left\{x+\mu, \sup _{\beta<Q(x)} x^{a}(\beta)\right\} .
$$

In other words, if there is no traffic at all, then the total time needed for the trip is

$$
\mu=\frac{[\text { length of the highway }]}{[\text { maximum speed }]}=\frac{T}{f^{\prime}(0)} .
$$

On the other hand, if the driver starting at time $x$ encounters traffic, his arrival time will simply be the supremum among the arrival times of all cars departed earlier.

Definition. We say that a bounded, nondecreasing initial data $Q(\cdot)$ satisfying (3.2) yields a Nash solution of the Cauchy problem (3.1)-(3.3) with initial and terminal cost functions $\varphi, \psi$ if there exists a constant $c$ such that:

(i) For almost every $\beta \in[0, \kappa]$ one has

$$
\varphi\left(x^{q}(\beta)\right)+\psi\left(x^{a}(\beta)\right)=c .
$$

(ii) For all $x \in \mathbb{R}$, there holds

$$
\varphi(x)+\psi(A(x)) \geq c .
$$




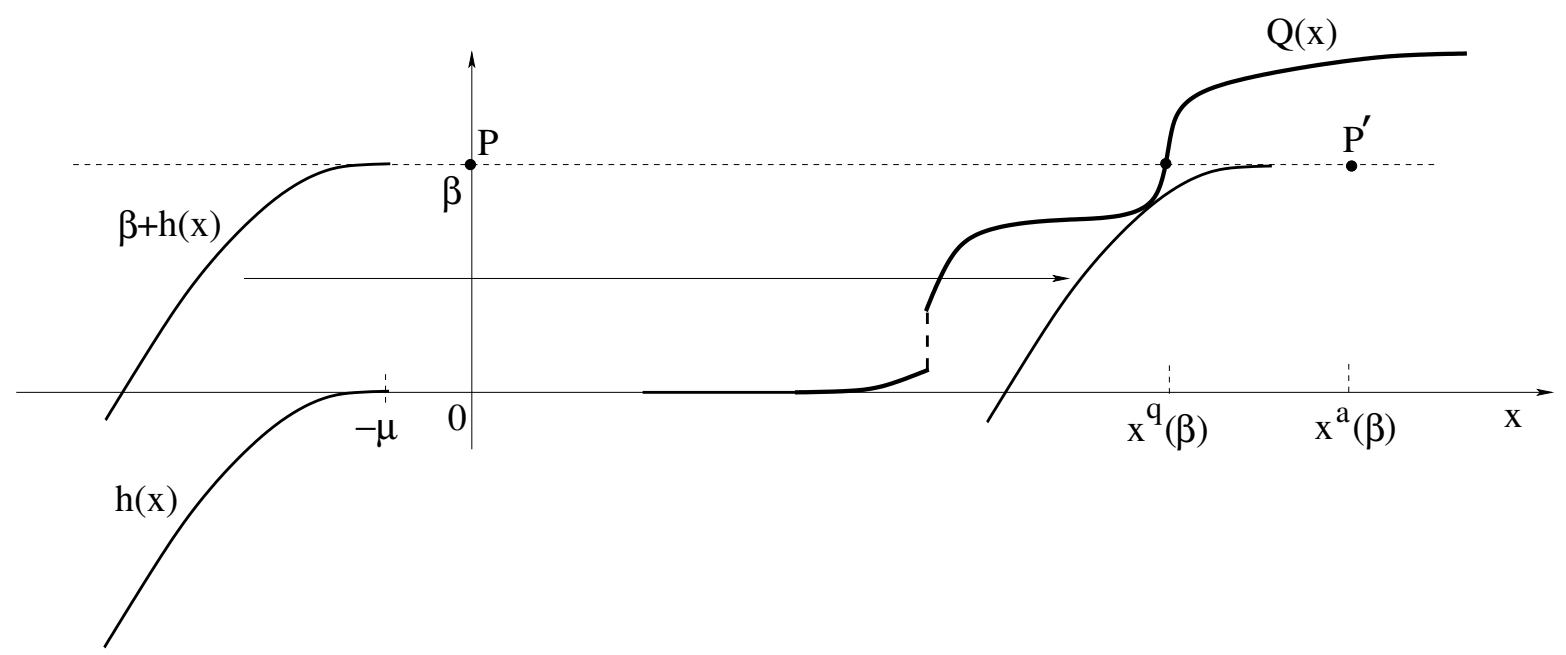

Figure 4: Given the function $Q(\cdot)$, let $\beta \in[0, Q(+\infty)]$ label a particular driver. The starting time $x^{q}(\beta)$ is then determined by the intersection of the graph of $Q$ with the line $Q=\beta$. The arrival time $x^{a}(\beta)$ is determined as follows: shift the graph of the function $x \mapsto \beta+h(x)$ horizontally, until it lies entirely below the graph of $Q$. In the figure, the size $\left|P^{\prime}-P\right|$ of this shift yields precisely the arrival time $x^{a}(\beta)$.

In connection with the traffic model, condition (i) states that all drivers bear the same cost $c$. Condition (ii) says that, regardless of the starting time $x$, no one can achieve a cost $<c$.

Theorem 3. Let the flux function $f$ and the initial and terminal cost functions $\varphi, \psi$ satisfy the assumptions (A1)-(A2). Moreover, assume that $f^{\prime}(u) \rightarrow+\infty$ as $u \rightarrow M-$.

Then, for every $\kappa>0$, the Cauchy problem (3.1), (3.3) admits a unique Nash equilibrium solution, with initial data $Q(\cdot)$ satisfying (3.2).

Proof. The result will be proved in several steps. The overall strategy is to show that

(i) To each $c \in \mathbb{R}$ there exists a unique Nash equilibrium having cost $c$. This is determined by some initial data $Q(\cdot)$ having total mass $\kappa(c) \doteq Q(+\infty)$.

(ii) For some minimum cost $c_{0}$, the map $c \mapsto \kappa(c)$ is a strictly increasing, continuous map from $\left[c_{0},+\infty[\right.$ onto $[0,+\infty[$.

1. For a fixed constant $c$, let $\mathcal{Q}_{c}$ be the family of all bounded, non-decreasing initial data $Q(\cdot)$ such that $Q(-\infty)=0$, and such that the corresponding solution $U=U(t, x)$ in (3.4) satisfies

$$
\varphi\left(x^{q}(\beta)\right)+\psi\left(x^{a}(\beta)\right) \leq c \quad \text { for a.e. } \beta \in[0, Q(+\infty)] .
$$

We claim that the maximum number of drivers

$$
\kappa(c) \doteq \sup \left\{Q(+\infty) ; Q \in \mathcal{Q}_{c}\right\}
$$

is finite. Indeed, by the assumptions (A2), there exists a bounded interval $\left[x^{-}, x^{+}\right]$such that

$$
\varphi(x)>c \quad \text { for all } x<x^{-}, \quad \varphi(x)+\psi(x)>c \quad \text { for all } x>x^{+} .
$$


By (3.15), this implies that, for every $Q \in \mathcal{Q}_{c}$, the corresponding function $\bar{U}$ in (3.3) must be constant on $\left.]-\infty, x^{-}\right]$and on $\left[x^{+},+\infty[\right.$. We thus obtain the bound

$$
\kappa(c)=\bar{U}(+\infty)=\int_{-\infty}^{+\infty} \bar{U}_{x}(x) d x \leq \int_{x^{-}}^{x^{+}} \bar{U}_{x}(x) d x \leq M\left(x^{+}-x^{-}\right) .
$$

2. In the remainder of the proof we show the initial data

$$
Q^{*}(x) \doteq \sup \left\{Q(x) ; \quad Q \in \mathcal{Q}_{c}\right\}
$$

yields a Nash equilibrium solution $U^{*}=U^{*}(t, x)$.

To begin with, we check that, if $Q_{1}, Q_{2} \in \mathcal{Q}_{c}$, then the function $Q_{3}(x) \doteq \max \left\{Q_{1}(x), Q_{2}(x)\right\}$ also lies in $\mathcal{Q}_{c}$. Indeed, let $\beta \in[0, \kappa(c)]$ and, to fix the ideas, assume

$$
x_{3}^{q}(\beta)=\min \left\{x_{1}^{q}(\beta), x_{2}^{q}(\beta)\right\}=x_{1}^{q}(\beta),
$$

with obvious meaning of notations. Then

$$
x_{3}^{a}(\beta) \leq \min \left\{x_{1}^{a}(\beta), x_{2}^{a}(\beta)\right\} \leq x_{1}^{a}(\beta) .
$$

Since $\varphi^{\prime}<0$ and $\psi^{\prime} \geq 0$, this yields

$$
\varphi\left(x_{3}^{q}(\beta)\right)+\psi\left(x_{3}^{a}(\beta)\right) \leq \varphi\left(x_{1}^{q}(\beta)\right)+\psi\left(x_{1}^{a}(\beta)\right) \leq c .
$$

Therefore, $Q_{3} \in \mathcal{Q}_{c}$ as well.

Using this property of the set $\mathcal{Q}_{c}$, we can construct a sequence functions $Q_{n}(\cdot) \in \mathcal{Q}_{c}$ such that, for every $x \in \mathbb{R}$, the sequence $Q_{n}(x)$ increases monotonically to $Q^{*}(x)$. Since all functions $Q_{n}$ are constant outside a bounded interval, we clearly have $Q^{*}(-\infty)=0, Q^{*}(+\infty)=\kappa(c)$. We claim that $Q^{*} \in \mathcal{Q}_{c}$. Indeed, by monotonicity and pointwise convergence we have $x_{n}^{q}(\beta) \rightarrow$ $x^{q}(\beta)$, for a.e $\beta \in[0, \kappa(c)]$. Therefore, $x_{n}^{a}(\beta) \rightarrow x^{a}(\beta)$ for a.e $\beta$. The continuity of $\varphi$ and $\psi$ now yields

$$
\varphi\left(x^{q}(\beta)\right)+\psi\left(x_{1}^{a}(\beta)\right) \leq c \quad \text { for a.e. } \beta \in[0, \kappa(c)] .
$$

Therefore, $Q^{*} \in \mathcal{Q}_{c}$.

3. In this step we show that the solution $U^{*}$ of (3.1) with initial data $Q^{*}$ is a Nash equilibrium.

To prove (3.13) we argue by contradiction. Suppose there exists some $\beta$ such that the points $x_{*}^{q}(\beta), x_{*}^{a}(\beta)$ are well defined (as in (3.10)-(3.11), with $Q, U$ replaced by $\left.Q^{*}, U^{*}\right)$, but

$$
\varphi\left(x_{*}^{q}(\beta)\right)+\psi\left(x_{*}^{a}(\beta)\right)<c .
$$

By the continuity of $\varphi$, we can choose $\varepsilon>0$ small enough so that

$$
\varphi\left(x_{*}^{q}(\beta)-\varepsilon\right)+\psi\left(x_{*}^{a}(\beta)\right)<c .
$$

Consider the perturbed initial data

$$
\widetilde{Q}(x) \doteq\left\{\begin{array}{cl}
\beta & \text { if } x \in\left[x_{*}^{q}(\beta)-\varepsilon, x_{*}^{q}(\beta)\right], \\
Q^{*}(x) & \text { otherwise. }
\end{array}\right.
$$


We claim that $\widetilde{Q} \in \mathcal{Q}_{c}$. Indeed, since $\widetilde{Q} \geq Q^{*}$, the corresponding solutions satisfy $\widetilde{U}(T, x) \geq$ $U^{*}(T, x)$ for every $x$. For any $\beta^{\prime} \in[0, \kappa(c)]$, two cases can arise:

CASE 1: $\quad x_{*}^{q}\left(\beta^{\prime}\right) \notin\left[x_{*}^{q}(\beta)-\varepsilon, x_{*}^{q}(\beta)\right]$. In this case one has

$$
\tilde{x}^{q}\left(\beta^{\prime}\right)=x_{*}^{q}\left(\beta^{\prime}\right), \quad \tilde{x}^{a}\left(\beta^{\prime}\right) \leq x_{*}^{a}\left(\beta^{\prime}\right) .
$$

Therefore, recalling that $\varphi^{\prime}<0$ while $\psi^{\prime} \geq 0$, we obtain

$$
\varphi\left(\tilde{x}^{q}\left(\beta^{\prime}\right)\right)+\psi\left(\tilde{x}^{a}\left(\beta^{\prime}\right)\right) \leq \varphi\left(x_{*}^{q}\left(\beta^{\prime}\right)\right)+\psi\left(x_{*}^{a}\left(\beta^{\prime}\right)\right) \leq c .
$$

CASE 2: $x_{*}^{q}\left(\beta^{\prime}\right) \in\left[x_{*}^{q}(\beta)-\varepsilon, x_{*}^{q}(\beta)\right]$. In this case, by (3.18) one has

$$
\begin{gathered}
\tilde{x}^{q}\left(\beta^{\prime}\right) \geq x_{*}^{q}(\beta)-\varepsilon, \quad \tilde{x}^{a}\left(\beta^{\prime}\right) \leq x_{*}^{a}\left(\beta^{\prime}\right) . \\
\varphi\left(\tilde{x}^{q}\left(\beta^{\prime}\right)\right)+\psi\left(\tilde{x}^{a}\left(\beta^{\prime}\right)\right) \leq \varphi\left(x_{*}^{q}(\beta)-\varepsilon\right)+\psi\left(x_{*}^{a}\left(\beta^{\prime}\right)\right)<c .
\end{gathered}
$$

We conclude that $\widetilde{Q} \in \mathcal{Q}_{c}$. Since $\widetilde{Q}(x)>Q^{*}(x)$ for $x_{*}^{q}-\varepsilon<x<x_{*}^{q}$, this contradicts the definition (3.17). Hence $Q^{*}$ satisfies the condition (3.13).

It remains to prove that (3.14) also holds. Let $x \in \mathbb{R}$ be given. Again we consider two cases.

CASE 1: There exists a sequence of values $\beta_{n} \rightarrow Q(x)$ satisfying (3.13), such that $x^{q}(\beta) \rightarrow x$. In this case we have $x^{a}\left(\beta_{n}\right) \rightarrow A(x)$. By the continuity of $\varphi, \psi$, the inequality (3.14) is then an immediate consequence of (3.13).

CASE 2: $Q(y)=\beta_{0}$ is a constant for all $y$ in a neighborhood of $x$. In this case, if

$$
A(x)=\inf _{\beta \rightarrow \beta_{0}+} x^{a}(\beta)
$$

we can still conclude

$$
\varphi(x)+\psi(A(x)) \geq \lim _{\beta \rightarrow \beta_{0}+}\left(\varphi\left(x^{q}(\beta)\right)+\psi\left(x^{a}(\beta)\right)\right)=c .
$$

It thus remains to examine the case where (3.19) does not hold. Assume that (3.14) fails. Then there exists $\delta>0$ such that

$$
A(x)+\delta \leq \inf _{\beta \rightarrow \beta_{0}+} x^{a}(\beta), \quad \varphi(x)+\psi(y) \leq c \quad \text { for all } y \in[A(x), A(x)+\delta] .
$$

Introduce the constant $\eta \doteq-h(-\mu-\delta)$ and consider the function

$$
\widetilde{Q}(y) \doteq\left\{\begin{array}{cc}
Q^{*}(y) & \text { if } y<x, \\
\max \left\{Q^{*}(y), \beta_{0}+\eta\right\} & \text { if } y \geq x
\end{array}\right.
$$

We claim that $\widetilde{Q} \in \mathcal{Q}_{c}$. Indeed, the choice of $\eta$ yields

$$
\begin{aligned}
& \beta<\beta_{0} \quad \Longrightarrow \quad \tilde{x}^{q}(\beta)=x_{*}^{q}(\beta), \quad \tilde{x}^{a}(\beta)=x_{*}^{a}(\beta), \\
& \beta_{0}<\beta<\beta_{0}+\eta \quad \Longrightarrow \quad \tilde{x}^{q}(\beta)=x, \quad \tilde{x}^{a}(\beta) \leq A(x)+\delta, \\
& \beta_{0}<\beta \quad \Longrightarrow \quad \tilde{x}^{q}(\beta)=x_{*}^{q}(\beta), \quad \tilde{x}^{a}(\beta)=x_{*}^{a}(\beta) .
\end{aligned}
$$


The above construction shows that, if (3.14) fails, then $Q^{*}$ is not maximal. This completes the proof that $Q^{*}$ provides a Nash equilibrium solution.

4. In this step we prove that, for any given $c \in \mathbb{R}$, the Nash equilibrium solution corresponding to the cost $c$ is unique.

For each $x \in \mathbb{R}$, denote by $z(x)$ the point such that

$$
\varphi(z(x))+\psi(x)=c .
$$

Notice that $z(x)$ is uniquely defined, because $\varphi$ is strictly decreasing. If now $Q$ is an initial data yielding a Nash solution $U=U(t, x)$ with cost $c$, recalling the definitions (3.9) one has

$$
x^{q}(\beta)=z\left(x^{a}(\beta)\right) \quad \text { for a.e. } \beta \in[0, Q(+\infty)] .
$$

Remark 5. Since the map $\beta \mapsto x^{a}(\beta)$ is strictly increasing, if $Q$ has a jump at a point $x_{0}$ then $\psi$ must be constant on the nontrivial interval $\left[x^{a}\left(Q\left(x_{0}-\right)\right), x^{a}\left(Q\left(x_{0}+\right)\right)\right]$. If we assume that the cost $\psi$ is strictly increasing, then the distribution $Q(\cdot)$ which yields a Nash equilibrium must be continuous.

Consider two initial data $Q_{1}(x)$ and $Q_{2}(x)$, yielding two Nash equilibrium solutions corresponding to the same cost $c$. Since $\varphi(s) \rightarrow+\infty$ as $s \rightarrow-\infty$, there exists $x^{-} \in \mathbb{R}$ such that $Q_{1}(x)=Q_{2}(x)=0$ for all $x \leq x^{-}+1$. The uniqueness property will be proved by showing that, for every $\varepsilon>0$,

$$
Q_{2}(x)-Q_{1}(x)<\varepsilon\left(x-x^{-}\right) \quad \text { for all } x>x^{-} .
$$

Indeed, if (3.22) fails, there exists a first point $x_{0}$ such that

$$
Q_{2}(x-)<Q_{1}(x-)+\varepsilon\left(x-x^{-}\right) \quad \text { for all } x<x_{0},
$$

and moreover: either

$$
Q_{2}\left(x_{0}-\right)=Q_{1}\left(x_{0}-\right)+\varepsilon\left(x_{0}-x^{-}\right),
$$

or else $Q_{2}$ has a jump at $x_{0}$ and

$$
Q_{2}\left(x_{0}-\right)<Q_{1}\left(x_{0}-\right)+\varepsilon\left(x_{0}-x^{-}\right) \quad Q_{2}\left(x_{0}+\right) \geq Q_{1}\left(x_{0}+\right)+\varepsilon\left(x_{0}-x^{-}\right) .
$$

A contradiction is derived as follows. Let $\beta \doteq Q_{1}\left(x_{0}-\right)$. Choose a point $\left(\xi, \beta^{\prime}\right)$ where the graph of the function $y \mapsto \beta+h\left(y-x_{1}^{a}(\beta)\right)$ touches the closure of the graph of $Q_{1}$. This means

$$
\beta^{\prime}=\beta+h\left(\xi-x_{1}^{q}(\beta)\right)=Q_{1}(\xi-) .
$$

We consider three cases.

CASE 1: (3.23)-(3.24) hold, with $\xi<x_{0}$ (see fig. 5, left).

Consider the higher level $\beta^{\prime \prime} \doteq \beta+\varepsilon\left(\xi-x^{-}\right)$and observe that $x_{2}^{a}\left(\beta^{\prime \prime}\right) \geq x_{1}^{a}(\beta)$. Since the map $x \mapsto z(x)$ defined at (3.20) is nondecreasing, this implies $z\left(x_{2}^{a}\left(\beta^{\prime \prime}\right)\right) \geq z\left(x_{1}^{a}(\beta)\right)=\xi$. Therefore $Q_{2}\left(x_{0}-\right) \leq Q_{2}\left(z\left(x_{2}^{a}\left(\beta^{\prime \prime}\right)\right)-\right)=\beta+\varepsilon\left(\xi-x^{-}\right)<\beta+\varepsilon\left(x_{0}-x^{-}\right)=Q_{1}\left(x_{0}-\right)+\varepsilon\left(x_{0}-x^{-}\right)$, 

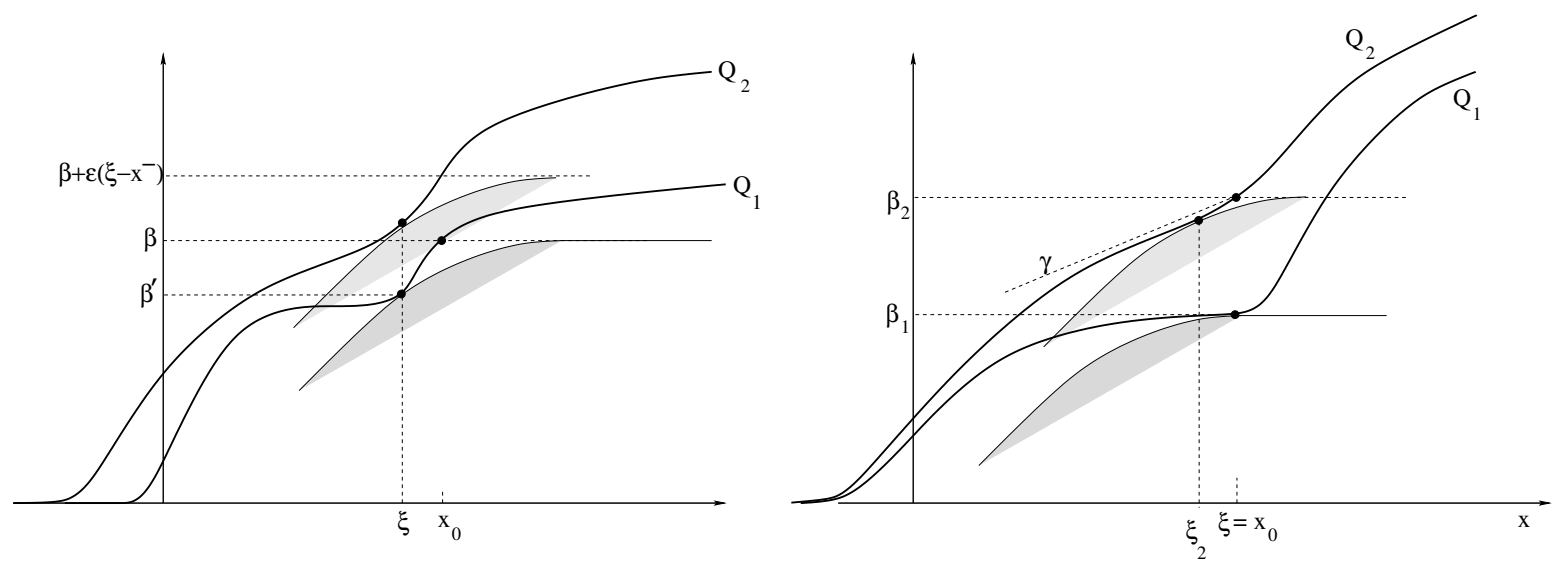

Figure 5: The construction used to prove uniqueness. Left: CASE 1. Right: CASE 2.

in contradiction with (3.24).

CASE 2: (3.23)-(3.24) hold, with $\xi=x_{0}$ (see fig. 5, right).

If $Q_{2}\left(x_{0}-\right)=\beta+\varepsilon\left(x_{0}-x^{-}\right)$, consider the line through the point $\left(x_{0}, \beta+\varepsilon\left(x_{0}-x^{-}\right)\right)$with slope $\varepsilon$, namely

$$
\gamma(x)=\beta+\varepsilon\left(x-x^{-}\right)
$$

By (3.23) it follows

$$
Q_{2}(x)<Q_{1}(x)+\varepsilon\left(x-x^{-}\right)<\gamma(x) \quad \text { for all } x \in\left[x^{-}, x_{0}[.\right.
$$

Observing that the function $h(\cdot)$ is continuously differentiable with

$$
\begin{array}{ll}
h^{\prime}(x)=0 & \text { if } x \geq-\mu, \\
h^{\prime}(x)>0 & \text { if } x<-\mu,
\end{array}
$$

the above inequality implies

$$
x_{2}^{a}\left(\beta+\varepsilon\left(x_{0}-x^{-}\right)\right)>A_{1}\left(x_{0}\right)=x_{0}+\mu .
$$

Indeed, setting $\beta_{2} \doteq Q_{2}\left(x_{0}-\right)$, the contact point $\xi_{2}$ defined by

$$
Q_{2}\left(\xi_{2}-\right)=\beta_{2}+h\left(\xi_{2}-x_{2}^{a}\left(\beta_{2}\right)\right)
$$

must satisfy $\xi_{2}<x_{0}$. By continuity, we can find $\beta^{\prime}<\beta+\varepsilon\left(x_{0}-x^{-}\right)$such that $x_{2}^{a}\left(\beta^{\prime}\right)>A_{1}\left(x_{0}\right)$. This implies

$$
Q_{2}\left(x_{0}-\right) \leq Q_{2}\left(z\left(x_{2}^{a}\left(\beta^{\prime}\right)\right)-\right)=\beta^{\prime}<\beta+\varepsilon\left(x_{0}-x^{-}\right),
$$

providing a contradiction.

CASE 3: the inequalities (3.23) and (3.25) hold.

In this case, define

$$
\beta_{1} \doteq Q_{1}\left(x_{0}-\right), \quad \eta \doteq \sup _{x<x_{0}}\left\{Q_{2}(x)-Q_{1}(x)\right\}<\varepsilon\left(x_{0}-x^{-}\right) .
$$



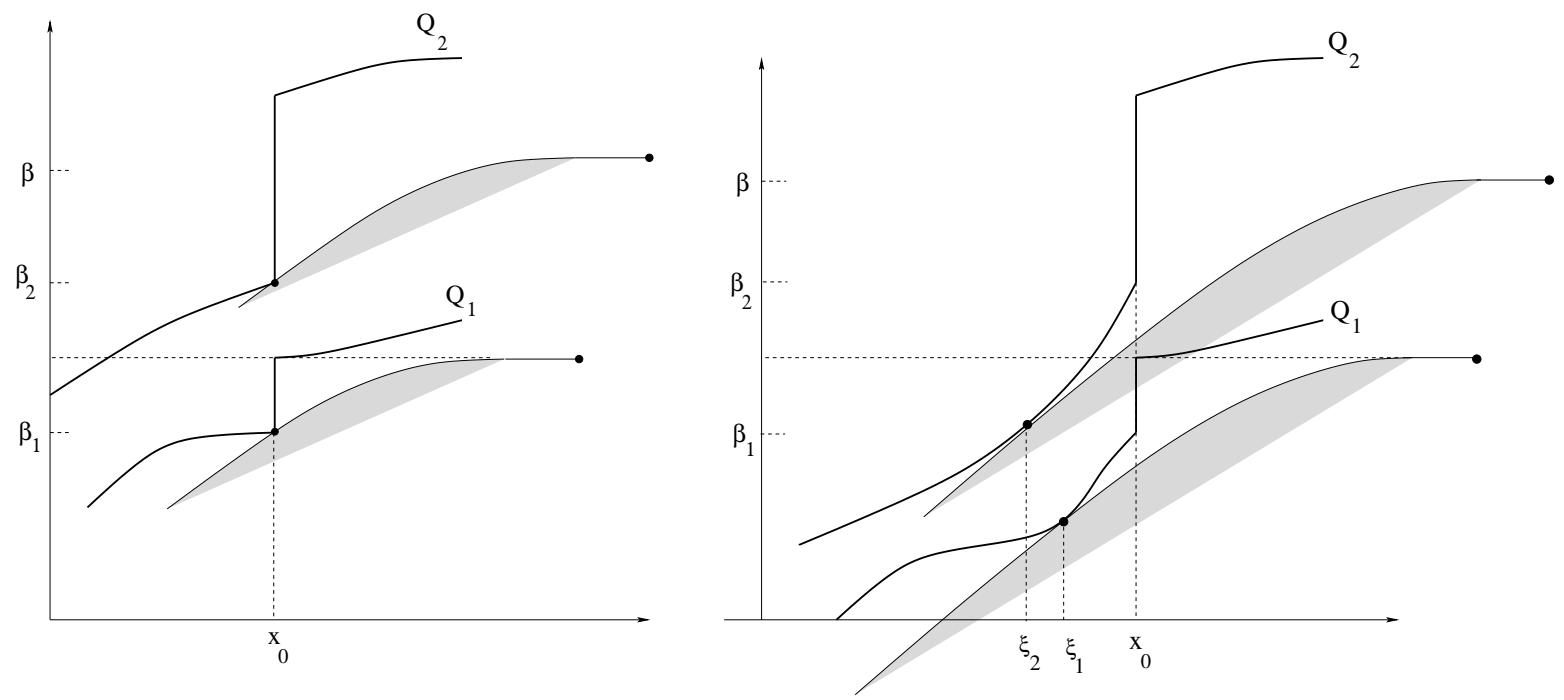

Figure 6: The construction used to prove uniqueness, in CASE 3. Here $P_{1}=\left(\beta_{1}, x_{1}^{a}\left(\beta_{1}\right)\right), P_{2}=$ $\left(\beta^{\prime}, x_{2}^{a}\left(\beta^{\prime}\right)\right)$. Since $\beta^{\prime}>\beta_{1}+\eta$, one has $x_{1}^{a}\left(\beta_{1}\right)<x_{2}^{a}\left(\beta^{\prime}\right)$.

Since

$$
Q_{2}(x) \leq Q_{1}(x)+\eta \quad \text { for all } x<x_{0}
$$

we have

$$
x_{2}^{a}(\beta+\eta) \geq x_{1}^{a}(\beta) \quad \text { for all } \beta<Q_{1}\left(x_{0}+\right) .
$$

Hence, for the distribution $Q_{1}$, the arrival time of a driver starting at a time $x>0$ satisfies

$$
\lim _{x \rightarrow x_{0}+} A_{1}(x) \leq x_{2}^{a}\left(\beta_{1}+\eta\right) .
$$

Choosing $\beta^{\prime}$ such that

$$
\beta_{1}+\eta<\beta^{\prime}<Q_{2}\left(x_{0}+\right),
$$

we achieve the strict inequality

$$
\lim _{x \rightarrow x_{0}+} A_{1}(x)<x_{2}^{a}\left(\beta_{1}+\eta\right) .
$$

The assumption that $Q_{2}$ is a Nash solution implies

$$
z\left(x_{2}^{a}\left(\beta^{\prime}\right)\right)=x_{0} .
$$

Thanks to (3.26), we can find $x>x_{0}$ such that

$$
\varphi(x)+\psi\left(A_{1}(x)\right)<\varphi\left(x_{0}\right)+\psi\left(x_{2}^{a}\left(\beta^{\prime}\right)\right)=c .
$$

Hence (3.14) fails, contradicting the assumption that $Q_{1}$ is a Nash equilibrium.

Putting together all cases, we thus obtain the uniqueness of the Nash equilibrium solution, for every fixed cost $c$.

5. By the previous steps, for each value $c$, there exists a unique Nash equilibrium solution with total mass $Q(+\infty)=\kappa(c)$, for some non-decreasing function $\kappa(\cdot)$. It is clear that $\kappa(c)=0$ for 
all $c$ sufficiently large and negative, while $\kappa(c) \rightarrow+\infty$ as $c \rightarrow+\infty$. Calling $c_{0} \doteq \inf \{c ; \kappa(c)>$ $0\}$, by the characterization of Nash solution (3.17) and by the continuity of the cost functions $\varphi, \psi$ it follows that $\kappa(\cdot)$ is strictly increasing on $\left[c_{0},+\infty[\right.$. To complete the proof, it remains to show that $\kappa(\cdot)$ is continuous, hence it maps $\left[c_{0},+\infty[\right.$ onto $[0,+\infty[$.

Observing that, for every $c$

$$
\mathcal{Q}_{c}=\bigcap_{c^{\prime}>c} \mathcal{Q}_{c^{\prime}}=\bigcap_{n \geq 1} \mathcal{Q}_{c+1 / n},
$$

it is clear that the map $c \mapsto \kappa(c)$ is right continuous. To show that

$$
\kappa(c)=\sup _{c^{\prime}<c} \kappa\left(c^{\prime}\right),
$$

we proceed as follows. Let $\varepsilon>0$ be given. Choose an initial data $Q \in \mathcal{Q}_{c}$ such that $Q(+\infty)=$ $\kappa(c)$. Consider the Lipschitz continuous function $\bar{U}$ as in (3.3) and, for some fixed $\varepsilon>0$, define

$$
\bar{U}_{\varepsilon}(x) \doteq(1-\varepsilon) \bar{U}(x) .
$$

Let $U, U_{\varepsilon}$ be the be the corresponding solutions of (3.1), given by the formula (3.4). Observe that, for a. e. $\beta \in[0, \kappa(c)]$ we have

$$
x^{d}(\beta) \doteq \inf \{x ; \bar{U}(x) \geq \beta\}=x_{\varepsilon}^{d}((1-\varepsilon) \beta) \doteq \inf \left\{x ; \bar{U}^{\varepsilon}(x) \geq(1-\varepsilon) \beta\right\} .
$$

We claim that, for a.e. $\beta \in[0, \kappa(c)]$ one has the strict inequality

$$
x^{a}(\beta) \doteq \inf \{x ; U(T, x) \geq \beta\}>x_{\varepsilon}^{a}((1-\varepsilon) \beta) \doteq \inf \left\{x ; U^{\varepsilon}(T, x) \geq(1-\varepsilon) \beta\right\} .
$$

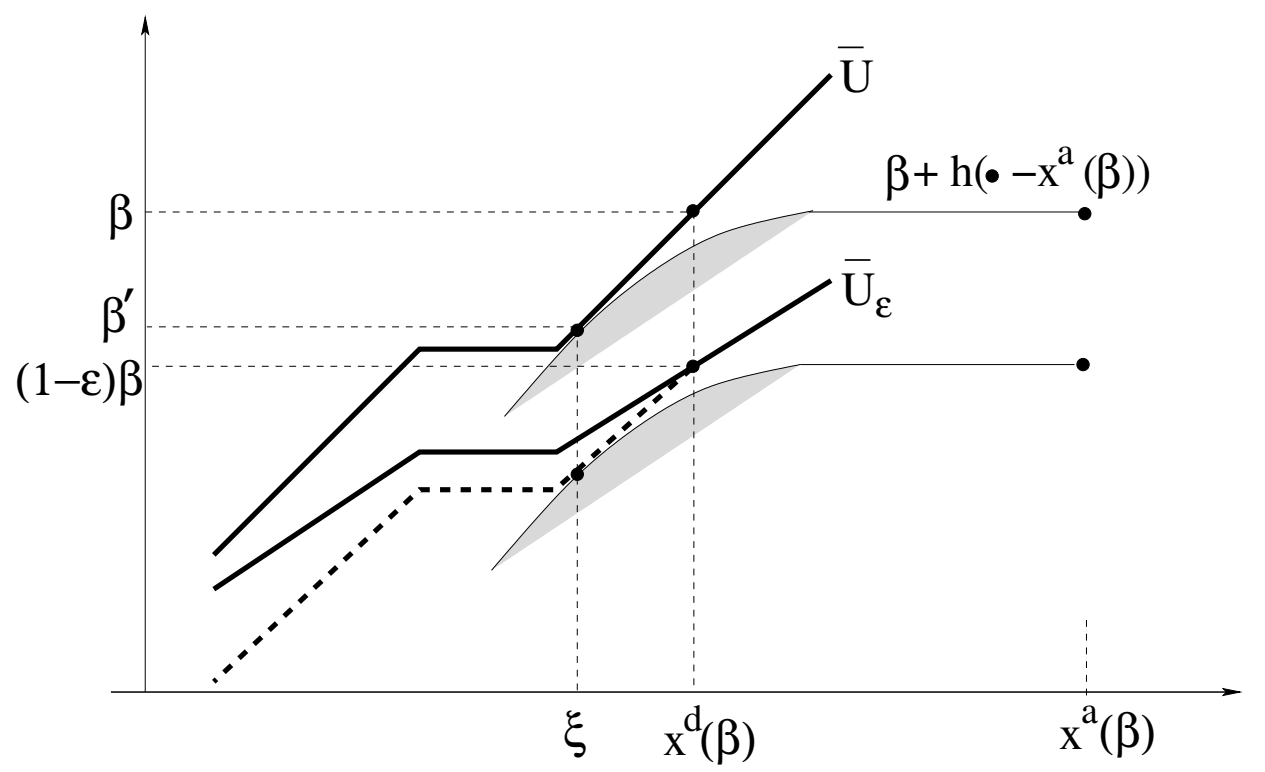

Figure 7: Proving the strict inequality $x_{\varepsilon}^{a}((1-\varepsilon) \beta)<x^{a}(\beta)$. For $y \leq x^{d}(\beta)$, the graph of the function $y \mapsto(1-\varepsilon) \beta+h\left(y-x^{a}(\beta)\right)$ lies strictly below the graph of $\bar{U}_{\varepsilon}$. Here the thick dotted polygonal denotes the graph of $y \mapsto \bar{U}(x)-\varepsilon \bar{U}\left(x^{d}(\beta)\right)$.

Indeed, for a.e. $\beta \in[0, \kappa(c)]$ there exist a unique point $x^{d}(\beta)$ such that

$$
\bar{U}\left(x^{d}(\beta)\right)=\beta, \quad \bar{U}_{x}\left(x^{d}(\beta)\right)>0 .
$$


For any $\beta$ as above, consider the arrival time

$$
x^{a}(\beta)=\inf \{x ; \beta+h(y-x) \leq \bar{U}(y) \text { for all } y \leq x\} .
$$

By the second relation in (3.30), any point $\left(\xi, \beta^{\prime}\right)$ where the graph of $\beta+h\left(\cdot-x^{a}(\beta)\right)$ touches the graph of $\bar{U}$ must satisfy

$$
\xi<x^{d}(\beta), \quad \beta^{\prime}<\beta .
$$

Moreover, for $y \leq x^{d}(\beta)$, one has

$$
(1-\varepsilon) \beta+h\left(y-x^{a}(\beta)\right) \leq \bar{U}(x)-\varepsilon \bar{U}\left(x^{d}(\beta)\right) .
$$

Therefore (see fig. 7), for $y \leq x^{d}(\beta)$, the graph of $y \mapsto(1-\varepsilon) \beta+h\left(y-x^{a}(\beta)\right.$ ) has strictly positive distance from the graph of $y \mapsto \bar{U}_{\varepsilon}(y)$. By the definition of $x_{\varepsilon}^{a}$, this yields the strict inequality (3.29).

For notational convenience, in the following we write $\beta_{\varepsilon} \doteq(1-\varepsilon) \beta$. In connection with the new initial data $\bar{U}_{\varepsilon}$, we claim that

$$
\varphi\left(x_{\varepsilon}^{q}\left(\beta_{\varepsilon}\right)\right)+\psi\left(x_{\varepsilon}^{a}\left(\beta_{\varepsilon}\right)\right)<c \quad \text { for a.e. } \beta_{\varepsilon} \in[0,(1-\varepsilon) \kappa(c)] .
$$

Indeed, since

$$
x_{\varepsilon}^{d}\left(\beta_{\varepsilon}\right)=x^{d}(\beta), \quad x^{a}(\beta)<x^{a}(\beta),
$$

two cases can arise.

CASE 1: $\psi\left(x_{\varepsilon}^{a}\left(\beta_{\varepsilon}\right)\right)<\psi\left(x^{a}(\beta)\right)$. Then we immediately conclude

$$
\varphi\left(x_{\varepsilon}^{q}\left(\beta_{\varepsilon}\right)\right)+\psi\left(x_{\varepsilon}^{a}\left(\beta_{\varepsilon}\right)\right)<\varphi\left(x^{q}(\beta)\right)+\psi\left(x^{a}(\beta)\right) \leq c .
$$

CASE 2: $\psi\left(x_{\varepsilon}^{a}\left(\beta_{\varepsilon}\right)\right)=\psi\left(x^{a}(\beta)\right.$. In this case we observe that, since $\psi$ is continuous and nondecreasing, there can be at most countably many disjoint open intervals $] a_{\ell}, b_{\ell}[$ such that $\psi$ is constant on each closed interval $J_{\ell} \doteq\left[a_{\ell}, b_{\ell}\right], \ell \geq 1$. Recalling that $\varphi$ is strictly decreasing, for each $\ell$ there can be at most one point $y_{\ell}$ such that

$$
\varphi\left(y_{\ell}\right)+\psi\left(a_{\ell}\right)=c
$$

Since the map $\beta \mapsto x^{d}(\beta)$ is strictly increasing, there can be at most one value $\beta_{\ell}$ such that $x^{d}\left(\beta_{\ell}\right)=y_{\ell}$.

If now $\psi\left(x_{\varepsilon}^{a}\left(\beta_{\varepsilon}\right)\right)=\psi\left(x^{a}(\beta)\right)$, then the two points $x^{a}(\beta), x_{\varepsilon}^{a}\left(\beta_{\varepsilon}\right)$ must lie in the same interval $J_{\ell}$, for some $\ell \geq 1$. Hence, either $\beta=\beta_{\ell}$, or else $\varphi\left(x^{d}(\beta)\right)+\psi\left(x^{a}(\beta)\right)<c$. The first possibility can occur only for countably many values of $\beta$. The second alternative leads to

$$
\varphi\left(x_{\varepsilon}^{q}\left(\beta_{\varepsilon}\right)\right)+\psi\left(x_{\varepsilon}^{a}\left(\beta_{\varepsilon}\right)\right) \leq \varphi\left(x^{q}(\beta)\right)+\psi\left(x^{a}(\beta)\right)<c .
$$

This proves our claim (3.31).

Based on the previous analysis, we can now choose $\delta>0$ such that the set

$$
B_{c-\delta} \doteq\left\{\beta \in[0,(1-\varepsilon) \kappa(c)] ; \varphi\left(x_{\varepsilon}^{q}(\beta)\right)+\psi\left(x_{\varepsilon}^{a}(\beta)\right)>c-\delta\right\}
$$


has measure

$$
\operatorname{meas}\left(B_{c-\delta}\right)<\varepsilon
$$

The new initial data

$$
\bar{U}^{\delta}(x) \doteq \int_{\left\{y \leq x ; \quad \bar{U}(y) \notin B_{c-\delta}\right\}} \bar{U}_{x}(y) d y
$$

satisfies

$$
\bar{U}^{\delta} \in \mathcal{Q}_{c-\delta}, \quad \bar{U}^{\delta}(+\infty) \geq(1-\varepsilon) \bar{U}(+\infty)-\varepsilon .
$$

Since $\varepsilon>0$ was arbitrary, this proves

$$
\kappa(c)=\sup _{c^{\prime}<c} \kappa\left(c^{\prime}\right) .
$$

Therefore the map $c \mapsto \kappa(c)$ is continuous, hence surjective. This completes the proof.

\section{Application to traffic flow}

In order to apply the previous results to our specific problem of traffic flow, it suffices to switch the variables $t, x$, and check that the flux function $f$, defined as a partial inverse of the map $\rho \mapsto \rho v(\rho)$, satisfies the assumptions (A1). Under natural hypotheses on the velocity $v=v(\rho)$, this is straightforward (see fig. 1). Indeed, define

$$
\rho^{*} \doteq \underset{\rho}{\operatorname{argmax}} \rho v(\rho), \quad M \doteq \rho^{*} v\left(\rho^{*}\right)=\max _{\rho} \rho v(\rho) .
$$

Assume that the second derivative of the flux of cars satisfies

$$
2 v^{\prime}(\rho)+\rho v^{\prime \prime}(\rho) \leq \gamma<0 \quad \text { for all } \rho \in\left[0, \rho^{*}\right] .
$$

Then the map $\rho \mapsto \rho v(\rho)$ is strictly increasing and concave on $\left[0, \rho^{*}\right]$. The inverse function $f:[0, M] \mapsto\left[0, \rho^{*}\right]$ is strictly increasing, uniformly convex, and satisfies $f^{\prime}(u) \rightarrow+\infty$ as $u \rightarrow M-$. Hence all the assumptions used in Theorems 1-3 hold.

In the next sections we explicitly compute the globally optimal solution and the Nash equilibrium solution for the traffic flow problem, in the case where the velocity function $v$ and the cost functions $\varphi, \psi$ are given by

$$
v(\rho)=\left(1-\frac{\rho}{\rho_{0}}\right) v_{0}, \quad \varphi(t)=-t, \quad \psi(t)= \begin{cases}0 & \text { if } t<0, \\ t^{2} & \text { if } t \geq 0 .\end{cases}
$$

\subsection{The globally optimal solution}

Consider the Cauchy problem

$$
\left\{\begin{array}{l}
u_{x}+f(u)_{t}=0 \\
u(t, 0)=\bar{u}(t)
\end{array} \quad t \in \mathbb{R}, \quad x \in[0, L],\right.
$$




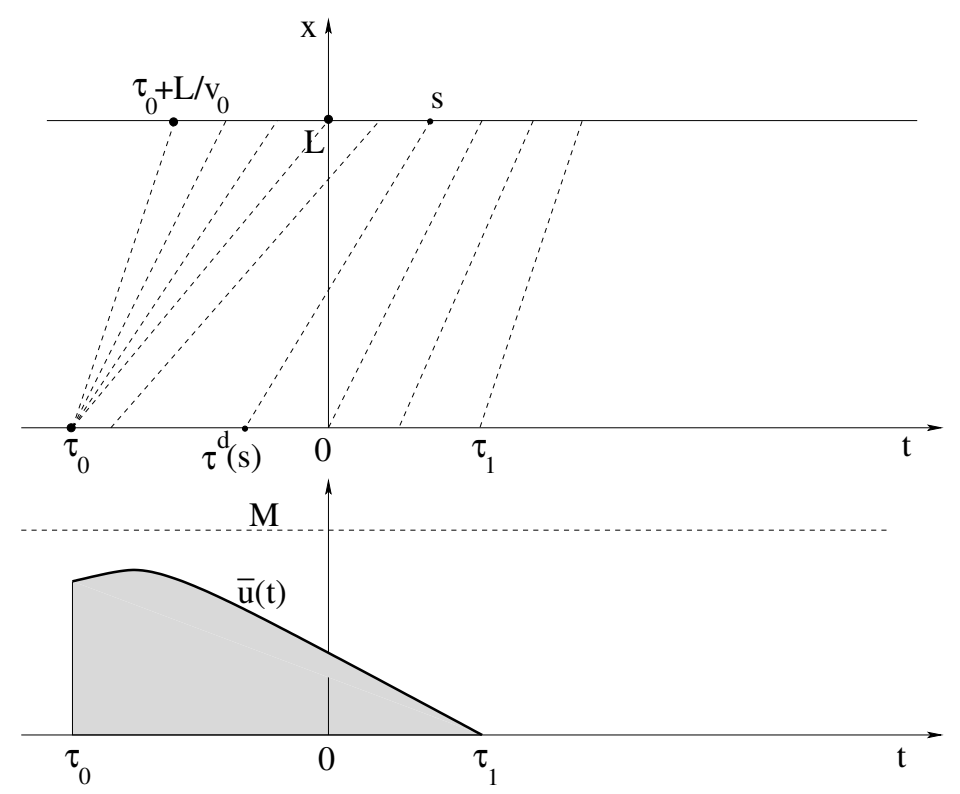

Figure 8: The globally optimal solution.

where $u=\rho v(\rho)$ and $f(u)=\rho$ is the inverse of the flux function in (1.3). We thus have

$$
\begin{array}{cl}
u=\rho v_{0}\left(1-\frac{\rho}{\rho_{0}}\right), \quad \rho^{*}=\frac{\rho_{0}}{2}, & M=\frac{\rho_{0} v_{0}}{4}, \\
f(u)=\rho=\frac{\rho_{0}}{2}\left(1-\sqrt{1-\frac{u}{M}}\right) & \rho \in[0, M], \\
f^{\prime}(u)=\frac{\rho_{0}}{4 M} \frac{1}{\sqrt{1-(u / M)}}, & \left(f^{\prime}\right)^{-1}(s)=M-\frac{\rho_{0}^{2}}{16 M s^{2}} .
\end{array}
$$

Observe that the speed of cars is $\leq v_{0}$. Hence the time needed to get to destination is $\geq L / v_{0}$. Setting

$$
c_{0} \doteq \min _{t \in \mathbb{R}}\left\{\varphi(t)+\psi\left(t+\frac{L}{v_{0}}\right)\right\}=\min _{t \in \mathbb{R}}\left\{-t+\left(t+\frac{L}{v_{0}}\right)^{2}\right\}=\frac{L}{v_{0}}-\frac{1}{4},
$$

it is clear that the total amount of cars that can incur in a cost $\leq c_{0}$ is zero.

Using Theorem 2, we now describe the globally optimal solution (2.33)-(2.34), corresponding to a cost $c>L / v_{0}>c_{0}$ (see fig. 8). Define the times

$$
\tau_{0} \doteq-c, \quad \tau_{1} \doteq \sup \left\{t ;-t+\left(t+\frac{L}{v_{0}}\right)^{2} \leq c\right\}=\frac{1}{2}-\frac{L}{v_{0}}+\sqrt{\frac{1}{4}+c-\frac{L}{v_{0}}} .
$$

Then the flux function $u=u(t, x)$ for the optimal solution is as follows (see fig. 8). To each terminal time $s \in\left[\tau_{0}+\frac{L}{v_{0}}, \tau_{1}+\frac{L}{v_{0}}\right]$ we can associate a unique initial time

$$
\tau^{q}(s)=\left\{\begin{array}{cl}
-c & \text { if } \quad s<0 \\
s^{2}-c & \text { if } \quad s \geq 0
\end{array}\right.
$$

so that

$$
\varphi\left(\tau^{q}(s)\right)+\psi(s)=c .
$$


At all points along the segment joining $\left(\tau^{q}(s), 0\right)$ with $(s, L)$, the flux function $u$ is given by

$$
u=\left(f^{\prime}\right)^{-1}\left(\frac{s-\tau^{q}(s)}{L}\right)
$$

so that $f^{\prime}(u)$ coincides with the slope of this segment. On the other hand,

$$
u(t, x)=0 \quad \text { if } t-\frac{x}{v_{0}} \notin\left[\tau_{0}, \tau_{1}\right] .
$$

Notice that the triangle with vertices $\left(\tau_{0}, 0\right),\left(\tau_{0}+\frac{L}{v_{0}}, L\right),(0, L)$ is the domain of a centered rarefaction wave. The initial data $\bar{u}=u(t, 0)$ is determined by

$$
\bar{u}\left(\tau^{q}(s)\right)=\left(f^{\prime}\right)^{-1}\left(\frac{s-s^{2}+c}{L}\right)=M-\frac{\rho_{0}^{2}}{16 M}\left(\frac{L}{s-s^{2}+c}\right)^{2} .
$$

Recalling that $t=\tau^{q}(s)=s^{2}-c$, we obtain

$$
\bar{u}(t)=\left\{\begin{array}{cc}
M-\frac{\rho_{0}^{2}}{16 M}\left(\frac{L}{\sqrt{c+t}-t}\right)^{2} & \text { if } t \in\left[\tau_{0}, \tau_{1}\right], \\
0 & \text { if } t \notin\left[\tau_{0}, \tau_{1}\right] .
\end{array}\right.
$$

Notice that the total flux

$$
\kappa(c)=\int_{\tau_{0}(c)}^{\tau_{1}(c)} M-\frac{\rho_{0}^{2}}{16 M}\left(\frac{L}{\sqrt{c+t}-t}\right)^{2} d t
$$

is a continuous, increasing function of $c$.

Remark 6. A globally optimal solution can be characterized by the identity (4.6), where $\tau^{q}(s)$ and $s$ represent the initial and terminal time along a characteristic. On the other hand, a Nash equilibrium solution can still be characterized by the identity (4.6), but with $\tau^{q}(s)$ and $s$ now being the initial and terminal time along a particle trajectory, i.e. the departure and arrival time of individual drivers. These are not at all the same. Indeed, characteristic curves for the conservation law in (4.2) satisfy the equation

$$
\frac{d t}{d x}=f^{\prime}(u)
$$

while particle trajectories satisfy

$$
\frac{d t}{d x}=\frac{1}{v}=\frac{\rho}{\rho v(\rho)}=\frac{f(u)}{u}<f^{\prime}(u) \quad \text { for } 0<u<M .
$$

The left hand sides of (4.8) and (4.9) coincide only in the limit as $u \rightarrow 0$.

\subsection{The Nash equilibrium solution}

We now construct a Nash equilibrium solution, where all drivers incur in the same cost $c$. The initial data will be described by the function $t \mapsto Q(t)$, counting the number of drivers that 
have started their journey (possibly joining the queue at the entrance of the highway, if there is any) within time $t$.

Fix any cost $c>L / v_{0}$ and let $\tau_{0}, \tau_{1}$ be as in (4.4). Moreover, let $s \mapsto \tau^{q}(s)$ be the map in (4.5). Let $\tilde{u}=\tilde{u}(t, x)$ be the solution to the Riemann problem

$$
\tilde{u}_{x}+f(\tilde{u})_{t}=0, \quad \tilde{u}(t, 0)= \begin{cases}0 & \text { if } t<\tau_{0}, \\ M & \text { if } t \geq \tau_{0} .\end{cases}
$$

Let

$$
\delta_{0} \doteq \int_{-\infty}^{0} \tilde{u}(t, L) d t>0
$$

be the total flux of this solution through the point $x=L$, for $t<0$. In other words, this is the total number of cars which arrive at destination before time $t=0$.
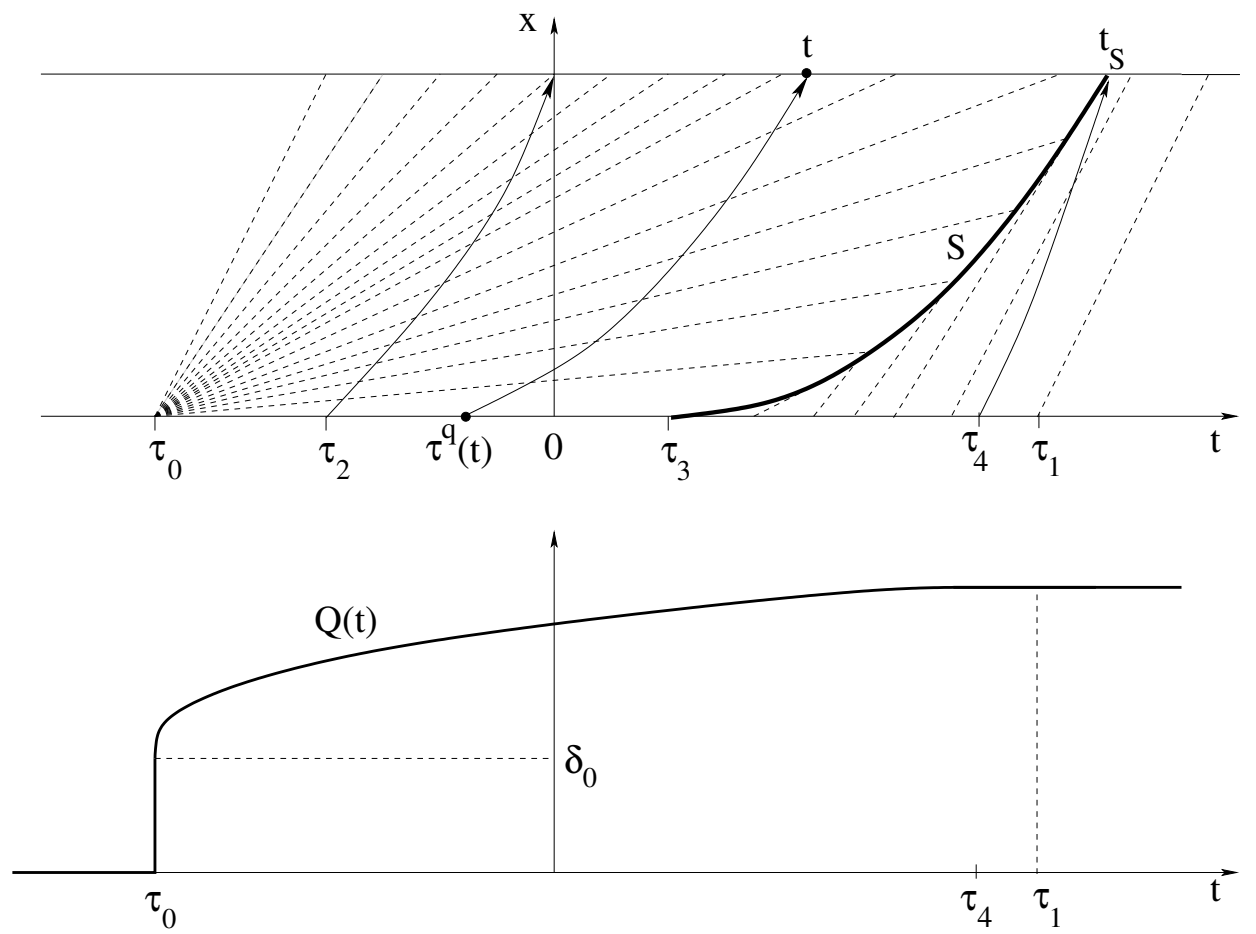

Figure 9: The Nash equilibrium solution. Here $\tau_{0}, \tau_{1}$ are the times defined at (4.4), while $\tau_{4}=\tau^{d}\left(t_{S}\right)$.

The Nash equilibrium solution has the following properties (see fig. 9).

- Before time $\tau_{0}$, no cars enter the queue.

- Exactly at time $\tau_{0}$, an amount $\delta_{0}$ of cars arrives and instantly forms a queue at the entrance of the highway.

- The last of the cars which entered the queue at $t=\tau_{0}$ departs at time $\tau_{2}=\tau_{0}+\left(\delta_{0} / M\right)$ and arrives at destination exactly at time $t=0$.

- The queue shrinks to zero at some time $\tau_{3}$. When this happens, a shock is formed, moving along some curve $S$. 
- After time $\tau_{3}$, cars keep coming to the entrance of the highway, and depart instantly, until time $\tau_{1}$. No driver begins his journey after time $\tau_{1}$.

To explicitly compute this solution, we first consider the problem

$$
\rho_{t}+\left(\rho v_{0}-\frac{\rho^{2} v_{0}}{\rho_{0}}\right)_{x}=0, \quad \rho(t, 0)= \begin{cases}0 & \text { if } t<\tau_{0}, \\ \rho_{0} / 2 & \text { if } t \geq \tau_{0} .\end{cases}
$$

Calling $\tilde{\rho}$ the solution of this Riemann problem, and setting $\tilde{u}=\tilde{\rho} v(\tilde{\rho})$, we find

$$
\tilde{\rho}(t, L)=\frac{\rho_{0}}{2}-\frac{L \rho_{0}}{2 v_{0}} \cdot \frac{1}{t-\tau_{0}}, \quad \tilde{u}(t, L)=M-\frac{L^{2} \rho_{0}}{4 v_{0}} \frac{1}{\left(t-\tau_{0}\right)^{2}}
$$

for $t \geq \tau_{0}+\left(L / v_{0}\right)$, while

$$
\tilde{\rho}(t, L)=\tilde{u}(t, L)=0 \quad \text { for } \quad t<\tau_{0}+\frac{L}{v_{0}} .
$$

This yields

$$
\delta_{0}=\int_{\tau_{0}+\left(L / v_{0}\right)}^{0} \tilde{u}(s, L) d s=-\frac{L^{2} \rho_{0}}{4 v_{0} \tau_{0}}-\frac{L \rho_{0}}{2}-M \tau_{0} .
$$

For $t>\tau_{0}$, the value of $Q(t)$ is computed using the equation

$$
Q\left(\tau^{q}(s)\right)-\delta_{0}=\int_{0}^{s} \tilde{u}(\xi, L) d \xi
$$

By (4.5) this yields

$$
Q(t)=\delta_{0}+\int_{0}^{\sqrt{t+c}} \tilde{u}(\xi, L) d \xi
$$

where $\tilde{u}$ was computed at (4.13). At the time $\tau_{3}$ such that

$$
Q\left(\tau_{3}\right)-M\left(\tau_{3}-\tau_{0}\right)=0,
$$

the queue is depleted and a shock is formed in the solution. Call $t_{S}$ the time where this shock hits the boundary $x=L$, and set $\tau_{4} \doteq \tau^{q}\left(t_{S}\right)$. Then for $t \in\left[\tau_{0}, \tau_{4}\right]$ the function $Q(\cdot)$ is still given by the explicit formula (4.15). On the other hand, for $\tau_{4}<t<\tau_{1}$, the value of $Q$ can be computed from the equation

$$
Q(t)=U(\sqrt{t+c}, L)
$$

where $U$ is the solution of (1.13). Since for $u>0$ the characteristic speed $f^{\prime}(u)$ is strictly larger than the particle speed $f(u) / u$, the right hand side of (4.17) can be computed in terms of the values of the initial data $Q$ on a strictly smaller interval, say $\left[\tau_{0}, t-\varepsilon\right]$.

\subsection{Some numerical results}

We first numerically compute a Nash equilibrium solution for the problem (4.1)-(4.2), with the following parameter values:

$$
L=1, \quad \rho_{0}=v_{0}=2, \quad M=\frac{\rho_{0} v_{0}}{4}=1, \quad \frac{L}{v_{0}}=\frac{1}{2},
$$


We start by choosing a common cost $c=2.7$ to each driver. Numerically, we find that this corresponds to a total flux $\kappa=\kappa(c)=3.80758$. The initial profile (4.15) is given by

$$
Q(t)=\left\{\begin{array}{cl}
0 & \text { if } t<-2.7, \\
1.7+\sqrt{t+2.7}+\frac{1}{4(\sqrt{t+2.7}+2.7)} & \text { if } \quad-2.7 \leq t \leq \tau_{4},
\end{array}\right.
$$

while $Q(t)=Q\left(\tau_{1}\right)$ is constant for $t \geq \tau_{1}$. Using (4.16), we find that the queue is depleted at time $\tau_{3}=0.9698$. For $t \in\left[\tau_{0}, \tau_{4}\right]$, the rate at which cars actually depart (after clearing the queue at the entrance of the highway) is given by

$$
u(t, 0)= \begin{cases}1 & \text { if } t \in[-2.7,0.9698], \\ \frac{1}{2 \sqrt{t+2.7}} \cdot\left(1-\frac{1}{4(\sqrt{t+2.7}+2.7)^{2}}\right) & \text { if } \left.t \in] 0.9698, \tau_{4}\right] .\end{cases}
$$

We use an upwind method to solve the conservation law and locate the position of the shock at the terminal point $x=1$. A numerical simulation shows that $t_{S}=2.055$, and therefore $\tau_{4}=t_{S}^{2}-2.7=1.5230$. The last driver begins his journey at time

$$
\tau_{1}=\sup \left\{t ; \quad-t+\left(t-\frac{1}{2}\right)^{2} \leq 2.7\right\}=1.5652 .
$$

The total cost of this Nash equilibrium solution is computed as

$$
J^{N a s h}=\int-t d Q(t)+\int \psi(t) \cdot u(1, t) d t=7.42913+2.8570=10.28613 .
$$

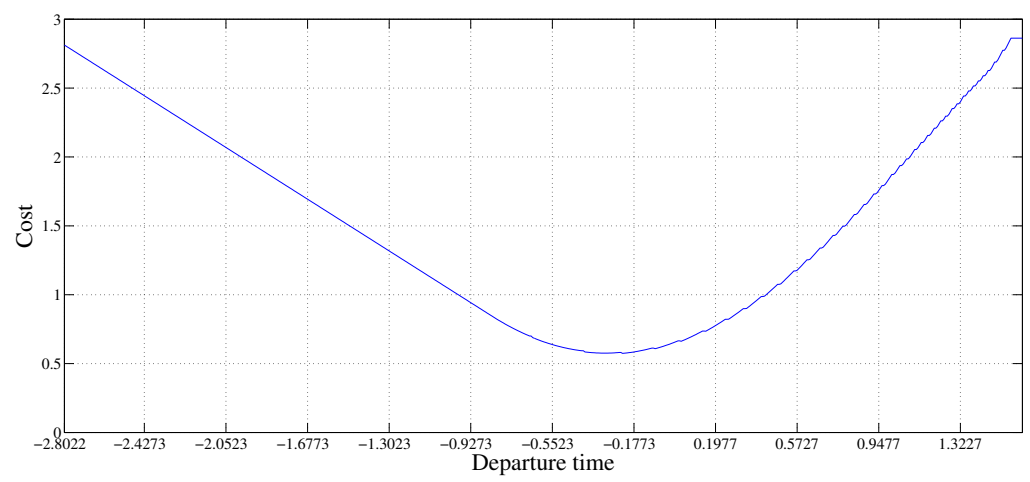

Figure 10: The cost to each driver as a function of the departure time $t$, in a globally optimal solution. By imposing the additional cost $\phi(t)=c_{\max }-c(t)$ at a toll booth at the entrance of the highway, the globally optimal solution becomes a Nash equilibrium.

Next, we compute the globally optimal solution for the same parameter values as in (4.18) and the same total flux $\kappa=3.80758$ found for the Nash solution. Numerically, we find that the solution is described by (4.7) with $c=2.80226$.

The initial flux for the globally optimal solution is

$$
u(t, 0)=\bar{u}(t)=\left\{\begin{array}{cl}
1-\frac{1}{4}\left(\frac{1}{\sqrt{2.80226+t}-t}\right)^{2} & \text { if } t \in[-2.80226,1.5976], \\
0 & \text { if } t \notin[-2.80226,1.5976] .
\end{array}\right.
$$


The flux at the terminal point of the highway $L=1$ can be explicitly computed as

$$
u(t, L)= \begin{cases}1-\frac{1}{4}\left(\frac{1}{t+2.80226}\right)^{2} & \text { if } t \in[-2.30226,0], \\ 1-\frac{1}{4}\left(\frac{1}{t-t^{2}+2.80226}\right)^{2} & \text { if } t \in] 0,2.0976] .\end{cases}
$$

The costs at $x=0$ (for starting early) and at $x=L$ (for arriving late) are computed by

$$
\int_{-2.80226}^{1.5976}-t \cdot u(t, 0) d t=3.03525, \quad \int_{0}^{2.0976} t^{2} u(t, L) d t=2.53612 .
$$

The total cost for this globally optimal solution is $J^{\text {min }}=5.57137$. Notice that this is much smaller than the total cost $J^{\text {Nash }}=10.28613$ for the Nash equilibrium solution.

It is interesting to compare the optimal solution in Fig. 8 with the "bang-bang" solution, where the flux of cars entering the highway at $x=0$ is either zero or maximum. Choosing the same parameters as in (4.18), and the same total flux $\kappa=3.80758$, the initial condition is now

$$
u^{b b}(t, 0)=\left\{\begin{array}{ll}
1 & \text { if } t \in\left[\tau_{2}, \tau_{3}\right], \\
0 & \text { if } t \notin\left[\tau_{2}, \tau_{3}\right],
\end{array} \quad \tau_{3}=\tau_{2}+\frac{\kappa}{M}=\tau_{2}+3.80758 .\right.
$$

The solution $u^{b b}$ with initial data (4.26) consists of a single centered rarefaction wave, bounded by a shock. In particular, the flux of cars at the end of the road, where $x=L$, is given by

$$
u^{b b}(t, L)=\left\{\begin{array}{cl}
1-\frac{1}{4}\left(\frac{1}{t-\tau_{2}}\right)^{2} & \text { if } t \in\left[\tau_{2}+1 / 2, t_{S}\right], \\
0 & \text { if } t \notin\left[\tau_{2}+1 / 2, t_{S}\right] .
\end{array}\right.
$$

Here $t_{S}$ denotes the position of the shock at $x=L$. This value can be easily computed using the conservation of the total flux: $\int u^{b b}(t, x) d t=\kappa$ for every $x \in[0, L]$. This yields

$$
t_{S}=\tau_{2}+\frac{1}{2}+4.255
$$

The optimal choice of $\tau_{2}$ is found to be

$$
\tau_{2}=\underset{\tau}{\operatorname{argmin}}\left\{\int_{\tau}^{\tau+3.8076}-t \cdot 1 d t+\int_{0}^{\tau+\frac{1}{2}+4.255} t^{2} \cdot u^{b b}(L, t) d t\right\}=-2.78836,
$$

yielding a total cost $J^{b b}=5.86767$. As expected, this is bigger than the cost $J^{\text {min }}=5.57137$ of the optimal solution.

\subsection{Optimal toll pricing}

As shown by the previous computations, the Nash equilibrium solution can be highly inefficient, yielding a total cost which is much higher than the globally optimal cost. A major goal of game theory is to devise incentives for the individual players, so that the Nash solution becomes more efficient, i.e. close to the globally optimal solution. 
In connection with traffic flow, a natural problem is the following. Assume that, by charging a (time dependent) toll $p(t)$ at the entrance of the highway, we wish to collect a total revenue $R$. What is the best way to choose the additional cost function $p(\cdot)$ ?

Notice that, if a flat rate $p(t) \equiv \bar{p}$ is imposed, each driver should thus be asked to pay $\bar{p}=R / \kappa$, where $\kappa$ is the total number of cars entering the highway. This choice, however, would not have any influence on the overall traffic pattern: replacing the starting cost $\varphi(t)$ with $\varphi(t)+\bar{p}$ does not change the Nash equilibrium solution.

On the other hand, by imposing a time dependent toll $p(\cdot)$, a more efficient traffic pattern can be achieved. This leads to the problem of finding a function $p(t) \geq 0$ which minimizes the total cost to all drivers

$$
\int(\varphi(t)+p(t)) u(t, 0) d t+\int \psi(t) u(t, L) d t
$$

subject to

$$
\int u(t, 0) d t=\kappa, \quad \quad \int p(t) u(t, 0) d t=R .
$$

Here $u=u(t, x)$ is the car flux, in the Nash equilibrium solution corresponding to the new starting cost $\tilde{\varphi}(t)=\varphi(t)+p(t)$ and the same arrival cost $\psi(t)$. Notice that, by Theorem 3 , the Nash solution is uniquely determined by the choice of $p(\cdot)$.

If the desired revenue $R>0$ is sufficiently large, this problem has a simple solution. Indeed, let $u=u^{*}(t, x)$ be the globally optimal solution to the problem (2.1)-(2.4). Let $\tau^{a}(t)$ be the arrival time of the driver who departs at time $t$, and let $c(t) \doteq \varphi(t)+\psi\left(\tau^{a}(t)\right)$ be his total cost. Call

$$
c_{\text {max }} \doteq \max \left\{c(t) ; \quad u^{*}(t, 0)>0\right\}
$$

the maximum cost among all drivers, in this globally optimal solution. Consider the toll fee $p(t) \doteq c_{R}-c(t)$, choosing the constant $c_{R}$ so that

$$
\int\left(c_{R}-c(t)\right) u^{*}(t, 0) d t=R
$$

If

$$
R \geq \int\left(c_{\max }-c(t)\right) u^{*}(t, 0) d t
$$

then one readily checks that $c_{R} \geq c_{\max }$ and the flux function $u^{*}$ provides a Nash solution for the traffic flow problem where the starting $\operatorname{cost} \varphi$ is replaced by $\tilde{\varphi}(t)=\varphi(t)+p(t)$. It is now clear that $p(\cdot)$ is the optimal pricing strategy, because it indices the most efficient traffic flow pattern, namely $u^{*}$.

Acknowledgement. This research was partially supported by NSF through grant EFRI1024707 "A theory of complex transportation network design".

\section{References}

[1] A. Bressan, Hyperbolic Systems of Conservation Laws. The One Dimensional Cauchy Problem, Oxford University Press, 2000. 
[2] A. Bressan, Noncooperative Differential Games. Milan J. of Mathematics,

[3] A. Bressan and A. Marson, A maximum principle for optimally controlled systems of conservation laws, Rend. Sem. Mat. Univ. Padova 94 (1995), 79-94.

[4] A. Bressan and W. Shen, Optimality conditions for solutions to hyperbolic balance laws, in "Control Methods in PDE - Dynamical Systems", F. Ancona, I. Lasieka, W. Littman, and R. Triggiani eds., AMS Contemporary Mathematics 426 (2007), 129-152.

[5] G. Crippa, C. Jimenez, and A. Pratelli, Optimum and equilibrium in a transport problem with queue penalization effect. Adv. Calc. Var. 2 (2009), 207246.

[6] L. C. Evans, Partial Differential Equations. Second edition. American Mathematical Society, Providence, RI, 2010.

[7] T. L. Friesz, Dynamic Optimization and Differential Games, Springer, New York, 2010.

[8] T. L. Friesz, T. Kim, C. Kwon, and M. A. Rigdon, Approximate network loading and dual-time-scale dynamic user equilibrium. Transportation Research Part B (2010).

[9] A. Fügenschuh, M. Herty, and A. Martin, Combinatorial and continuous models for the optimization of traffic flows on networks. SIAM J. Optim. 16 (2006), 1155-1176.

[10] M. Garavello and B. Piccoli, Traffic Flow on Networks. Conservation Laws Models. AIMS Series on Applied Mathematics, Springfield, Mo., 2006.

[11] M. Gugat, M. Herty, A. Klar, and G. Leugering, Optimal control for traffic flow networks. J. Optim. Theory Appl. 126 (2005), 589-616.

[12] M. Herty, C. Kirchner, and A. Klar, Instantaneous control for traffic flow. Math. Methods Appl. Sci. 30 (2007), 153-169.

[13] P. D. Lax, Hyperbolic systems of conservation laws II. Comm. Pure Appl. Math. 10 (1957), 537-566.

[14] P. Le Floch, Explicit formula for scalar non-linear conservation laws with boundary condition. Math. Models Appl. Sci. 10 (1988), 265-287.

[15] M. Lighthill and G. Whitham, On kinematic waves. II. A theory of traffic flow on long crowded roads. Proceedings of the Royal Society of London: Series A, 229 (1955), 317345 .

[16] P. I. Richards, Shock waves on the highway, Oper. Res. 4 (1956), 42-51.

[17] J. Smoller, Shock waves and reaction-diffusion equations. Second edition. SpringerVerlag, New York, 1994.

[18] S. Ulbrich, A sensitivity and adjoint calculus for discontinuous solutions of hyperbolic conservation laws with source terms, SIAM J. Control Optim. 41 (2002), 740-797.

[19] S. Ulbrich, Adjoint-based derivative computations for the optimal control of discontinuous solutions of hyperbolic conservation laws. Systems $\&$ Control Letters 48 (2003), 313-328. 